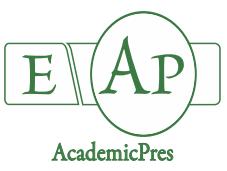

Safder I et al. (2021)

Notulae Botanicae Horti Agrobotanici Cluj-Napoca

Volume 49, Issue 3, Article number 12346

DOI: $10.15835 /$ nbha49312346

Research Article

\title{
Identification of SNPs in rice GPAT genes and in silico analysis of their functional impact on GPAT proteins
}

\section{Imran SAFDER ${ }^{a}$, Gaoneng SHAO ${ }^{\mathrm{b}}$, Zhonghua SHENG, Peisong HU, Shaoqing TANG*}

State Key Laboratory of Rice Biology and China National Center for Rice Improvement, China National Rice Research Institute, Hangzhou310006,China; imransafdar82@gmail.com; shaogaoneng@caas.cn; shengzhonghua@caas.cnhupeisong@caas.cn; shaoqingtangcnrri@126.com (" ${ }^{*}$ corresponding author)

${ }^{a, b}$ These authors contributed equally to the work

\begin{abstract}
SNPs are the most common nucleotide variations in the genome. Functional SNPs in the coding region, known as nonsynonymous SNPs (nsSNPs), change amino acid residues and affect protein function. Identifying functional SNPs is an uphill task as it is difficult to correlate between variation and phenotypes in association studies. Computational in silico analysis provides an opportunity to understand the SNPs functional impact to proteins and facilitate experimental approaches in understanding the relationship between the phenotype and genotype. Advancement in sequencing technologies contributed to sequencing thousands of genomes. As a result, many public databases have been designed incorporating this sequenced data to explore nucleotide variations. In this study, we explored functional SNPs in the rice GPAT family (as a model plant gene family), using 3000 Rice Genome Sequencing Project data. We identified 1056 SNPs, among hundred rice varieties in 26 GPAT genes, and filtered $98 \mathrm{nsSNPs}$. We further investigated the structural and functional impact of these nsSNPs using various computational tools and shortlisted 13 SNPs having high damaging effects on protein structure. We found that rice GPAT genes can be influenced by nsSNPs and they might have a major effect on regulation and function of GPAT genes. This information will be useful to understand the possible relationships between genetic mutation and phenotypic variation, and their functional implication on rice GPAT proteins. The study will also provide a computational pathway to identify SNPs in other rice gene families.
\end{abstract}

Keywords: 3000 Rice Genome project; functional SNPs; in silico analysis; nucleotide variation

\section{Introduction}

Rice genome was initially sequenced among cereal crops. It paved the path to sequence other complex plant genomes. The impact of sequencing rice genome was immediate: elicited high citations, DNA marker usage and research groups curated public databases (Jackson, 2016). An easy access to genome data stored in public databases provides opportunity to identify and explore nucleotide variations.

Received: 18 Apr 2021. Received in revised form: 22 Aug 2021. Accepted: 13 Sep 2021. Published online: 27 Sep 2021.

From Volume 49, Issue 1, 2021, Notulae Botanicae Horti Agrobotanici Cluj-Napoca journal uses article numbers in place of the traditional method of continuous pagination through the volume. The journal will continue to appear quarterly, as before, with four annual numbers. 
SNPs are the key nucleotide variations found predominantly in genome, and are simplest in form. For instance, in the human genome, $90 \%$ of the variations belong to SNPs, located after every $100-300 \mathrm{bp}$, and 170 bp in rice, but their densities may vary in different regions (Goff et al., 2002; Nelson et al., 2004; Kharabian, 2010). They have a wide presence in the genome and can be found in any part such as intronic region, mRNA, or in the intergenic regions.

SNPs are involved in many processes like the occurrence of disease in humans where a single nucleotide variation can be responsible for life-threatening disease (Lek et al., 2016). In plants they can induce physiological, biochemical and phenotypic changes and alter function (Gailing et al., 2009; Majeed et al., 2019; Sandhu et al., 2020). SNPs are named and categorized according to the region they are present and functions they perform. Among them, SNPs in the coding regions, termed as non-synonymous SNPs (nsSNPs) or missense SNPs are particularly important as they are responsible to change amino acid residues. These nsSNPs, therefore influence protein function, provide harmful or neutral effects to the protein structure, or reduce protein solubility (Zhang et al., 2018). They can alter gene regulation, protein charge, or either change inter and intra protein interactions. Therefore, they are crucial and it is beneficial to catalogue functional SNPs in different species. Although nsSNPs are important but SNPs including the coding synonymous SNPs (SNPs present in coding regions, but don't change the amino acid residues) or SNPs positioned outside the coding regions still have functions. They are attributed to impact gene expression due to changes in regulatory elements, exonic splice enhancers, binding of a transcription factor or in splicing processes (De Alencar and Lopes, 2010).

With the passage of time SNPs are gradually taking the place of SSRs, to use as markers in breeding purposes, as they are stable, efficient, have high presence, and bear less cost (McCouch et al., 2010; Mammadov et al., 2012). Marker-assisted selection is one of the methods used to attain molecular markers, discovered in large scale SNP-genotyping. Similarly, GWAS studies are also used to establish relationships with the SNP and the candidate genes (Zhang et al., 2018).

Identification of SNPs is an initial yet tedious task to explore functional effects of SNPs and their correlation with the phenotype(Tibbs Cortes et al., 2021), as it needs multi testing of hundreds of SNPs in the gene of interest. Still, the question would be which SNP set required for selection is important for association studies success, providing the strong reason for SNP variation. Many SNP assays have been employed, but because of not having accurate phenotypic and genotypic data, it's not easy to conduct these experiments. A particular breeding segregation population or near-isogenic line would be required to identify SNPS functional effects (Pea et al., 2013).

Similarly, another task after the explosion of genome wide studies is to understand functional significance of the identified SNP, and apply it to application studies. Regarding experimental assays, many SNPs from the GWAS are not as impactful to select for certain traits, and breeders found it difficult to use these molecular markers. As a successful breaded crop depend largely on the accuracy of these functional SNPs (Tibbs Cortes et al., 2021).

SNPs detected by experimental studies provide the strongest evidence to demonstrate functional significance. However, because of the lack of exactness of the phenotypic and genotypic data, these experiments are not easy to identify. An alternative, to explore the possible significant pattern of SNPs is to prioritize SNPs on their functional significance using in silico computational tools. These computational tools can identify and differentiate the neutral SNPs from the functionally significant SNPs, and infer nature of mutation and changes in protein structure caused by the particular SNP (Kharabian, 2010; Arshad and Attya Bhatti, 2018). In addition, similar to past in silico studies, they could also provide an independent evidence source alongside experimental studies to explore functionally important SNPs (Yang et al., 2004; Jiang et al., 2010; Chaisan et al., 2012; Bhardwaj et al., 2016; Withana et al., 2020).

Advances in big data analytics and artificial intelligence systems provide opportunities to build machine learning models that can predict accurate DNA variations and protein models identified from sequence data. For instance, Alphafold recently gained appreciation among the scientific community. Alphafold is an artificial 
intelligence and deep learning based prediction model for protein structures that achieved high accuracy even for sequences having lower homologous sequences (Senior et al., 2020; Yang et al., 2020). Such breakthroughs reveal the potential of AI on genome data and it is presumed that computational approaches will be frequently used in plants to recruit SNPs in the future (Korani et al., 2019).

There are several public databases to curate SNPs in rice genome. 3000 Rice Genome Project (3KRGP) consortium has sequenced 3000 rice varieties from 89 different countries to explore genomic diversity in rice crop, and approximately 18.9 million SNPs were identified from this project (Li et al., 2014). The consortium provides rice breeders and scientists a massive resource. In the past years, some comprehensive databases have been created using the 3KRGP data including, SNP-Seek database (Alexandrov et al., 2015), Rice Functional and Genomic Breeding (RFGB) (Wang et al., 2020a), RiceVarMap (Zhao et al., 2015). These public datasets provide opportunities to identify large-scale discovery of genomic variants associated with various traits, and can be tapped to increase yield potential. SNP-Seek database platform has incorporated millions of variants from 3000 rice accessions and provide easy access to mine alleles (Mansueto et al., 2016).

In this study, we aim to analyze all the functional SNPs in the rice Glycerol-3-phosphate acyltransferase (GPAT) gene family, using $3 \mathrm{~K}$ RGP data among 100 Chinese rice varieties from different geographical regions. We used rice GPAT genes as a model family, we previously identified in a genome wide study (Safder et al., 2021) to demonstrate in silico analysis in a gene family. We used different computational tools to explore SNPs, and their impact to the structure and function on rice GPAT proteins and prioritized SNPs having significant impact on GPAT proteins. The study will provide useful information about important SNPs that can affect protein functions and would be useful in future investigations.

\section{Materials and Methods}

\section{SNP Dataset and missense SNP identification in GPATgenes}

We retrieved the SNP data set of 100 rice accessions from the SNP-Seek database (http://snpseek.irri.org) (Alexandrov et al., 2015) at each rice GPAT locus. We used 26 GPAT locus positions identified in a genome wide study (Table S6). Then, we searched each locus position among 100 rice varieties in the SNPSeek database and find all the SNPs in 26 GPAT genes. Afterwards, we filtered all missense or nonsynonymous SNPs (nsSNPs) among initially identified SNPs. We find and documented nsSNPs details (SNP position, protein remnant change) to investigate further.

\section{SNPs identification with damaging effects}

We used three computational databases to explore functional impact of nsSNPs on protein structures including, Protein Variation Effect Analyzer (PROVEAN) [http://provean.jcvi.org/index.php] (Choi et al., 2012; Choi and Chan, 2015), Sorting Intolerant from Tolerant (SIFT) (Ng and Henikoff, 2003), and PolyPhen-2 (Adzhubei et al., 2010). These computational tools predicts whether an amino acide substitution by a DNA variant affects protein function based on sequence homology and physical properties of amino acids. The nsSNPs having a damaging or deleterious effect identified by these three tools were regarded as high risk nsSNPs and taken further for more investigation to analyze their putative effect.

\section{$S N P$ s influence on structural and functional properties of GPAT proteins}

$G P A T$ protein sequences carrying the high risk nsSNPs identified in the previous step were further examined along with the mutated amino acid residues, and submitted to the MutPred v1.2 (Li et al., 2009a) database. The MutPred database investigates the outcome of mutations at proteins and predicts the molecular mechanism associated with the mutation and also provides different gain and loss structural properties of a protein. 


\section{Effects on protein stability}

We used I-Mutant to examine protein stability affected by nonsynonymous SNPs by submitting the normal and mutated amino acid sequences. I-Mutant evaluates protein stability changes or any structural change after variation. The tool provides a relativity RI index (RI) of results, ranges 0 to 10 score, showing the reliability of the score.

\section{Evolutionary conservation of amino acid positions}

We used ConSurf tool to determine amino acids evolutionary conservation, affected by nsSNPs; to analyze if this amino acid position is highly conserved in a protein sequence. Consurf analyzes the degree of evolutionary conservation using 50 homologous sequences from different species based on phylogenetic relation. We considered those positions important, if they were highly conserved and located on sites affected by nsSNPs. (Ashkenazy et al., 2010; Celniker et al., 2013; Ashkenazy et al., 2016).

\section{$3 D$ modeling of protein structures and RMSD calculation}

Native and mutated protein structure models were generated using protein homology tools to evaluate effects to protein structure caused by high risk nsSNPs. Phyre2 (Kelley et al., 2015) was used to generate the protein models, and these structures were further viewed by Chimera(Pettersen et al., 2004). In addition, we used two more tools including Root Mean Square Deviation (RMSD), and Template Modeling Score (TMalign). RMSD indicates variation between two protein structures; a high score shows more variation between native and mutant structure (Carugo and Pongor, 2001; Zhang and Skolnick, 2005).

\section{Post translational modification (PTM) sites}

Post translation modification sites (PTM) in the rice GPAT Proteins were predicted including the phosphorylation, ubiquitylation and methylation sites. We used two computational tools to predict every PTM event. Phosphorylation PTM sites at Serine (S), Threonine (T), and Tyrosine $(\mathrm{Y})$ amino acid residues were predicted using GPS 5.0 (Xue et al., 2005) and NetPhos 3.1 (Blom et al., 1999). In NetPhos 3.1, residues having at least 0.5 score were considered as phosphorylated. Ubiquitylation PTM sites were predicted using BDMPUB (Li et al., 2009b) and UbPred (Radivojac et al., 2010). In UbPred only those residues were considered having a 0.62 or more score. Methlyation sites were predicted using PSSMe (Wen et al., 2016) and iMethylPseAAC (Qiu et al., 2014) tools. PSSMe predicted those lysine or arginine residues that had higher probability ratios.

\section{Results}

Functional SNPs extracted from the rice SNP-seek database

We used the rice SNP-seek database to explore all the SNPs in 26 GPAT genes. We used rice GPAT gene family as a model plant gene family, we reported in a genome wide study (Safder et al., 2021). SNP-seek database comprised 18 million SNPs identified in 3KRGP. To recruit SNPs, we shortlisted 100 Chinese rice accessions from different geographical regions (including indica, japonica) (Table S1). Initially we find 1056 SNPs, among 100 varieties at each GPAT locus; 99 were found in the UTR regions, 683 in the intronic regions and 140 in the codon synonymous region (Figure 1). We find 98 SNPs (non-synonymous SNPs or nsSNPs) in the coding regions, which can influence protein structure and function (Table 1). We selected these 98 nsSNPs for further investigation. 
Safder I et al. (2021). Not Bot Horti Agrobo 49(3):12346

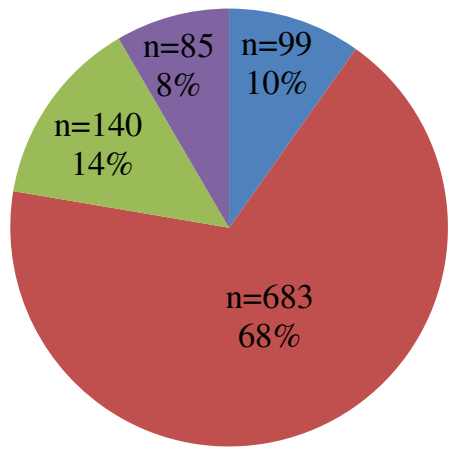

- UTR SNPs

- Intron SNPs

- Codon Synonymous SNPs

- Codon non synonymous SNPs

Figure 1. SNPs percentage in 26 rice GPATgenes

SNPs in different regions, including the UTR region, intronic region, and coding synonymous and nonsynonymous region

Table 1. Detailed information of 98 nsSNPs in 26 GPAT genes

\begin{tabular}{|c|c|c|c|}
\hline Gene ID & SNP position & Allele change & Amino acid position and residue change \\
\hline \multirow{4}{*}{ OsGPAT1 } & 8348616 & $\mathrm{G}>\mathrm{T}$ & Ala242Ser \\
\hline & 8350199 & $C>G$ & Ala362Gly \\
\hline & 8350403 & $\mathrm{G}>\mathrm{A}$ & Arg430His \\
\hline & 8350709 & $\mathrm{C}>\mathrm{T}$ & $\mathrm{Ala} 532 \mathrm{Val}$ \\
\hline \multirow{3}{*}{ OsGPAT2 } & 10970025 & $\mathrm{G}>\mathrm{C}$ & Thr223Ser \\
\hline & 10972698 & $\mathrm{C}>\mathrm{T}$ & Glu62Lys \\
\hline & 10972857 & $\mathrm{C}>\mathrm{T}$ & Gly9Ser \\
\hline OsGPAT4 & 25257427 & $\mathrm{~T}>\mathrm{C}$ & Phe59Ser \\
\hline \multirow{3}{*}{ OsGPAT5 } & 33154556 & $\mathrm{C}>\mathrm{A}$ & Gly348Cys \\
\hline & 33156296 & $\mathrm{C}>\mathrm{A}$ & Met88Ile \\
\hline & 33156464 & $\mathrm{C}>\mathrm{A}$ & Lys32Asn \\
\hline OsGPAT6 & 3686407 & $\mathrm{G}>\mathrm{A}$ & Ala255Val \\
\hline \multirow{5}{*}{ OsGPAT7 } & 40865354 & $A>G$ & Val452Ala \\
\hline & 40865364 & $\mathrm{~T}>\mathrm{C}$ & Asn449Asp \\
\hline & 40865933 & $\mathrm{~T}>\mathrm{C}$ & Gly292Gly \\
\hline & 40867989 & $\mathrm{~A}>\mathrm{G}$ & Val81Ala \\
\hline & 40868136 & $\mathrm{G}>\mathrm{A}$ & Ala32Val \\
\hline \multirow{4}{*}{ OsGPAT8 } & 776045 & $\mathrm{C}>\mathrm{T}$ & Val406Met \\
\hline & 776197 & $\mathrm{C}>\mathrm{G}$ & Arg355Pro \\
\hline & 776230 & $\mathrm{G}>\mathrm{A}$ & Ala344Val \\
\hline & 778246 & $\mathrm{C}>\mathrm{T}$ & Gly8Glu \\
\hline OsGPAT9 & 30152069 & $\mathrm{C}>\mathrm{A}$ & Gln361His \\
\hline \multirow{3}{*}{ OsGPAT 10} & 34985769 & $\mathrm{G}>\mathrm{A}$ & Met160Ile \\
\hline & 34985895 & $\mathrm{G}>\mathrm{C}$ & Gln202His \\
\hline & 34985957 & $\mathrm{G}>\mathrm{A}$ & Gly223Asp \\
\hline \multirow{7}{*}{ OsGPAT11 } & 31786785 & $\mathrm{~T}>\mathrm{C}$ & Val45Ala \\
\hline & 31787035 & $\mathrm{G}>\mathrm{C}$ & Lys99Asn \\
\hline & 31787058 & $A>G$ & Asn 107Ser \\
\hline & 31787320 & $C>G$ & His160Asp \\
\hline & 31787530 & $A>G$ & Asp191Gly \\
\hline & 31787808 & $C>G$ & Asp252Glu \\
\hline & 31787824 & $A>G$ & Arg258Gly \\
\hline OsGPAT12 & 34058002 & $\mathrm{G}>\mathrm{A}$ & Ala34Val \\
\hline \multirow{3}{*}{ OsGPAT 13} & 11754042 & $\mathrm{G}>\mathrm{A}$ & Ala357Val \\
\hline & 11755949 & $\mathrm{C}>\mathrm{A}$ & Ala127Ser \\
\hline & 11756221 & $\mathrm{G}>\mathrm{T}$ & Thr36Lys \\
\hline \multirow{3}{*}{ OsGPAT 16} & 22481879 & $\mathrm{G}>\mathrm{A}$ & Ala51Thr \\
\hline & 22484156 & $\mathrm{C}>\mathrm{A}$ & Gln207Lys \\
\hline & 22483271 & $\mathrm{G}>\mathrm{C}$ & Ala502Pro \\
\hline OsGPAT 17 & 24725803 & $\mathrm{C}>\mathrm{T}$ & Pro157Leu \\
\hline
\end{tabular}


Safder I et al. (2021). Not Bot Horti Agrobo 49(3):12346

\begin{tabular}{|c|c|c|c|}
\hline \multirow{2}{*}{ OsGPAT 18} & 30123014 & $\mathrm{G}>\mathrm{C}$ & Asp36His \\
\hline & 30127549 & $\mathrm{G}>\mathrm{C}$ & Gly545Arg \\
\hline \multirow{4}{*}{ OsGPAT 19} & 20809659 & $\mathrm{C}>\mathrm{T}$ & Ser231Asn \\
\hline & 20810625 & $\mathrm{~T}>\mathrm{G}$ & Met122Leu \\
\hline & 20811166 & $\mathrm{~T}>\mathrm{C}$ & Ile90Val \\
\hline & 20812394 & $\mathrm{G}>\mathrm{C}$ & Pro15Ala \\
\hline \multirow{9}{*}{ OsGPAT20 } & 1757860 & $\mathrm{C}>\mathrm{T}$ & Gly447Ser \\
\hline & 1757860 & $\mathrm{G}>\mathrm{T}$ & Phe412Leu \\
\hline & 1758474 & $\mathrm{G}>\mathrm{A}$ & His399Tyr \\
\hline & 1758474 & $A>G$ & Val242Ala \\
\hline & 1758624 & $\mathrm{G}>\mathrm{A}$ & Ser161Ile \\
\hline & 1758717 & $\mathrm{C}>\mathrm{A}$ & Ala155Val \\
\hline & 1758774 & $\mathrm{G}>\mathrm{A}$ & Ala142Val \\
\hline & 1758815 & $\mathrm{G}>\mathrm{A}$ & Phe128Leu \\
\hline & 1758876 & $\mathrm{C}>\mathrm{A}$ & Arg108Leu \\
\hline OsGPAT22 & 22060049 & $\mathrm{G}>\mathrm{C}$ & Ala258Pro \\
\hline \multirow{5}{*}{ Os GPAT24 } & 25200311 & $\mathrm{~T}>\mathrm{C}$ & Gln327Arg \\
\hline & 25200336 & $\mathrm{C}>\mathrm{T}$ & Ala319Thr \\
\hline & 25201064 & $\mathrm{G}>\mathrm{A}$ & .Pro297Leu \\
\hline & 25201089 & $\mathrm{C}>\mathrm{A}$ & Gly289Cys \\
\hline & 25201303 & $\mathrm{C}>\mathrm{T}$ & Ala256Thr \\
\hline \multirow{37}{*}{ OsGPAT25 } & 27486970 & $\mathrm{C}>\mathrm{T}$ & Pro2Leu \\
\hline & 27486991 & $\mathrm{G}>\mathrm{A}$ & Arg9Lys \\
\hline & 27487044 & $\mathrm{~T}>\mathrm{C}$ & Ser27Pro \\
\hline & 27487108 & $\mathrm{~T}>\mathrm{C}$ & Phe48Ser \\
\hline & 27487132 & $\mathrm{C}>\mathrm{T}$ & Ala56Val \\
\hline & 27487269 & $\mathrm{G}>\mathrm{A}$ & Val102Ile \\
\hline & 27487390 & $\mathrm{~A}>\mathrm{T}$ & Glu142Val \\
\hline & 27487401 & $\mathrm{G}>\mathrm{A}$ & Asp146Asn \\
\hline & 27487501 & $\mathrm{C}>\mathrm{T}$ & Ser179Leu \\
\hline & 27487577 & $\mathrm{G}>\mathrm{C}$ & Glu204Asp \\
\hline & 27487578 & $\mathrm{G}>\mathrm{A}$ & Val205Met \\
\hline & 27487604 & $\mathrm{G}>\mathrm{T}$ & Leu213Phe \\
\hline & 27487627 & $\mathrm{C}>\mathrm{T}$ & Thr221Ile \\
\hline & 27489105 & $\mathrm{G}>\mathrm{A}$ & Arg242His \\
\hline & 27489194 & $\mathrm{G}>\mathrm{A}$ & Ala272Thr \\
\hline & 27489200 & $\mathrm{G}>\mathrm{A}$ & Gly274Ser \\
\hline & 27489701 & $\mathrm{G}>\mathrm{C}$ & Asp441His \\
\hline & 27489766 & $\mathrm{C}>\mathrm{C}$ & Ile462Met \\
\hline & 27489853 & $\mathrm{C}>\mathrm{C}$ & Asp491Glu \\
\hline & 27489854 & $\mathrm{G}>\mathrm{A}$ & Val492Met \\
\hline & 27489880 & $\mathrm{G}>\mathrm{T}$ & Met500Ile \\
\hline & 27489956 & $\mathrm{G}>\mathrm{C}$ & Val526Leu \\
\hline & 27489979 & $\mathrm{C}>\mathrm{G}$ & Asn533Lys \\
\hline & 27489986 & $\mathrm{G}>\mathrm{G} / \mathrm{T}$ & Ala536Ser \\
\hline & 23091766 & $\mathrm{C}>\mathrm{G}$ & Ala44Gly \\
\hline & 23091780 & $A>G$ & Thr49Ala \\
\hline & 23091784 & $\mathrm{~T}>\mathrm{G}$ & Val50Gly \\
\hline & 23091790 & $\mathrm{~T}>\mathrm{C}$ & Met52Thr \\
\hline & 23091918 & $\mathrm{G}>\mathrm{C}$ & Val95Leu \\
\hline & 23091937 & $\mathrm{G}>\mathrm{C}$ & Gly101Ala \\
\hline & 23091943 & $\mathrm{G}>\mathrm{C}$ & Gly103Ala \\
\hline & 23092023 & $\mathrm{~A}>\mathrm{G}$ & Thr130Ala \\
\hline & 23092134 & $\mathrm{G}>\mathrm{A}$ & Ala167Thr \\
\hline & 23092180 & $\mathrm{~A}>\mathrm{T}$ & Tyr182Phe \\
\hline & 23092335 & $\mathrm{G}>\mathrm{A}$ & Val234Ile \\
\hline & 23093266 & $\mathrm{C}>\mathrm{T}$ & Pro268Ser \\
\hline & 23094109 & $\mathrm{G}>\mathrm{A}$ & Val549Ile \\
\hline
\end{tabular}




\section{Deleterious nsSNPs explored from Computational tools}

By employing in silico tools, SIFT, PROVEAN and PolyPhen, we explored 98 nsSNPs effect to variant amino acid residues. These tools predict functional effects of amino acid substitutions to the protein structure (Table 2).

Each tool has a cut-off value; a nsSNP is considered functional below this value (Table 2.). For instance, PROVEAN score is -2.5, below this cutoff, the substitutions are considered "deleterious", having high risk or deleterious effects on protein, and above this cutoff variant are considered Neutral. We considered nsSNPs as high risk or having damaging effects, if predicted by 2 of these tools. We find at least 13 nsSNPs (Table 2) in different genes having high risk or damaging effects on the protein structure.

Table 2. Effect of nsSNPs on protein structures analyzed by different computational tools

\begin{tabular}{|c|c|c|c|c|c|c|}
\hline \multirow{2}{*}{$\begin{array}{c}\text { Aminoacid } \\
\text { change }\end{array}$} & \multicolumn{2}{|c|}{ SIFT } & \multicolumn{2}{|c|}{ PROVEAN } & \multicolumn{2}{|l|}{ Polyphen-2 } \\
\hline & $\begin{array}{c}\text { SIFT } \\
\text { prediction }\end{array}$ & $\begin{array}{c}\text { SIFT } \\
\text { tolerance }\end{array}$ & Score & Cutoff $(-2.5)$ & Effect & Score \\
\hline Ala242Ser & Tolerated & 0.88 & 0.708 & Neutral & Benign & \\
\hline Ala362Gly & Tolerated & 0.37 & -0.054 & Neutral & Benign & \\
\hline Arg430His & Affected & 0.05 & -1.533 & Neutral & Probably damaging & 0.999 \\
\hline Ala532Val & Tolerated & 1 & -3.007 & Deleterious & Possibly damaging & 0.915 \\
\hline Thr223Ser & Tolerated & 0.44 & -0.186 & Neutral & Benign & \\
\hline Glu62Lys & Tolerated & 0.06 & -3.674 & Deleterious & Probably damaging & 0.997 \\
\hline Gly9Ser & Tolerated & 0.62 & -0.835 & Neutral & Possibly damaging & 0.762 \\
\hline Phe59Ser & Tolerated & 0.42 & 0.462 & Neutral & Benign & \\
\hline Gly348Cys & Tolerated & 0.12 & -1.308 & Neutral & Benign & \\
\hline Met88Ile & Tolerated & 0.39 & -0.138 & Neutral & Benign & \\
\hline Lys32Asn & Affected & 0 & -1.235 & Neutral & Possibly damaging & 0.625 \\
\hline Ala255Val & Tolerated & 0.18 & 1.378 & Neutral & Benign & \\
\hline Val452Ala & Affected & 0 & -0.456 & Neutral & Possibly damaging & 0.889 \\
\hline Asn449Asp & Affected & 0 & 0.192 & Neutral & Benign & \\
\hline Gly292Gly & Affected & 0 & 0 & Neutral & Benign & \\
\hline Val81Ala & Affected & 0 & 0.18 & Neutral & Benign & \\
\hline Ala32Val & Affected & 0 & -0.578 & Neutral & Probably damaging & 0.998 \\
\hline Val406Met & Tolerated & 0.51 & 0.981 & Neutral & Benign & \\
\hline Arg355Pro & Affected & 0 & -6.543 & Deleterious & Probably damaging & 1 \\
\hline Ala344Val & Tolerated & 0.23 & 2.209 & Neutral & Benign & \\
\hline Gly8Glu & Tolerated & 1 & 0.107 & Neutral & Probably damaging & 1 \\
\hline Gln361His & Tolerated & 0.46 & -1.184 & Neutral & Possibly damaging & 0.513 \\
\hline Met160Ile & Tolerated & 0.41 & -0.922 & Neutral & Benign & \\
\hline Gln202His & Tolerated & 0.2 & -0.086 & Neutral & Benign & \\
\hline Gly223Asp & Tolerated & 0.26 & -0.506 & Neutral & Probably damaging & 0.997 \\
\hline Val45Ala & Tolerated & 0.31 & -0.73 & Neutral & Benign & \\
\hline Lys99Asn & Tolerated & 0.07 & -0.922 & Neutral & Benign & \\
\hline Asn107Ser & Tolerated & 0.72 & -1.117 & Neutral & Benign & \\
\hline His160Asp & Tolerated & 1 & 5.701 & Neutral & Benign & \\
\hline Asp191Gly & Tolerated & 1 & 6.58 & Neutral & Benign & \\
\hline Asp252Glu & Tolerated & 0.8 & -0.411 & Neutral & Benign & \\
\hline Arg258Gly & Tolerated & 0.28 & -1.074 & Neutral & Benign & \\
\hline Ala34Val & Tolerated & 0.09 & -0.907 & Neutral & Possibly damaging & 0.62 \\
\hline
\end{tabular}


Safder I et al. (2021). Not Bot Horti Agrobo 49(3):12346

\begin{tabular}{|c|c|c|c|c|c|c|}
\hline Ala357Val & Tolerated & 1 & -0.489 & Neutral & Benign & \\
\hline Ala127Ser & Tolerated & 0.7 & -1.866 & Neutral & Probably damaging & 0.997 \\
\hline Thr36Lys & Tolerated & 1 & -0.455 & Neutral & Benign & \\
\hline Ala51Thr & Tolerated & 0.07 & -0.77 & Neutral & Possibly damaging & 0.863 \\
\hline Gln207Lys & Tolerated & 0.97 & -0.497 & Neutral & Possibly damaging & 0.78 \\
\hline Ala502Pro & Affected & 0 & -0.911 & Neutral & Probably damaging & 0.997 \\
\hline Pro157Leu & Affected & 0 & -8.92 & Deleterious & Probably damaging & 1 \\
\hline Asp36His & Affected & 0 & -0.25 & Neutral & Probably damaging & 0.988 \\
\hline Gly545Arg & Tolerated & 0.08 & 0.216 & Neutral & Possibly damaging & 0.641 \\
\hline Ser231Asn & Tolerated & 0.11 & 1.647 & Neutral & Benign & \\
\hline Met122Leu & Tolerated & 1 & -0.159 & Neutral & Benign & \\
\hline Ile90Val & Tolerated & 0.63 & -0.503 & Neutral & Benign & \\
\hline Pro15Ala & Tolerated & 1 & 0.036 & Neutral & Benign & \\
\hline Gly447Ser & Affected & 0 & -4.122 & Deleterious & Probably damaging & 1 \\
\hline Phe412Leu & Tolerated & 0.73 & -2.128 & Neutral & Benign & \\
\hline His399Tyr & Tolerated & 1 & -1.996 & Neutral & Possibly damaging & 0.748 \\
\hline Val242Ala & Tolerated & 0.15 & -1.585 & Neutral & Benign & \\
\hline Ser161Ile & Tolerated & 0.27 & -0.482 & Neutral & Probably damaging & 0.984 \\
\hline Ala155Val & Tolerated & 1 & 1.86 & Neutral & Benign & \\
\hline Ala142Val & Tolerated & 0.34 & -0.772 & Neutral & Benign & \\
\hline Phe128Leu & Affected & 0 & -5.526 & Deleterious & Probably damaging & 0.984 \\
\hline Arg108Leu & Tolerated & 0.08 & -1.221 & Neutral & Possibly damaging & 0.458 \\
\hline Ala258Pro & Tolerated & 0.2 & 2.652 & Neutral & Benign & \\
\hline Gln327Arg & Tolerated & 0.16 & -0.172 & Neutral & Benign & \\
\hline Ala319Thr & Tolerated & 0.39 & -0.591 & Neutral & Benign & \\
\hline Pro297Leu & Tolerated & 0.27 & -2.273 & Neutral & Possibly damaging & 0.548 \\
\hline Gly289Cys & Affected & 0.01 & -4.878 & Deleterious & Probably damaging & 0.999 \\
\hline Ala256Thr & Tolerated & 0.06 & 0.161 & Neutral & Benign & \\
\hline Pro2Leu & Affected & 0 & 0.883 & Neutral & Benign & \\
\hline Arg9Lys & Affected & 0 & -0.021 & Neutral & Benign & \\
\hline Ser27Pro & Affected & 0 & -0.847 & Neutral & Possibly damaging & 0.777 \\
\hline Phe48Ser & Affected & 0 & -0.213 & Neutral & Benign & \\
\hline Ala56Val & Tolerated & 0.61 & -1.302 & Neutral & Possibly damaging & 0.938 \\
\hline Val102Ile & Tolerated & 0.81 & 0.474 & Neutral & Benign & \\
\hline Glu142Val & Tolerated & 0.41 & 1.225 & Neutral & Benign & \\
\hline Asp146Asn & Tolerated & 0.2 & -2.508 & Deleterious & Possibly damaging & 0.752 \\
\hline Ser179Leu & Tolerated & 0.58 & 2.299 & Neutral & Benign & \\
\hline Glu204Asp & Tolerated & 0.63 & -0.936 & Neutral & Possibly damaging & 0.593 \\
\hline Val205Met & Affected & 0.04 & -0.176 & Neutral & Possibly damaging & 0.503 \\
\hline Leu213Phe & Tolerated & 0.7 & -0.862 & Neutral & Benign & \\
\hline Thr221Ile & Tolerated & 0.2 & -2.353 & Neutral & Benign & \\
\hline Arg242His & Tolerated & 0.14 & -0.896 & Neutral & Benign & \\
\hline Ala272Thr & Tolerated & 1 & 4.744 & Neutral & Benign & \\
\hline Gly274Ser & Tolerated & 0.35 & 1.037 & Neutral & Benign & \\
\hline Asp441His & Tolerated & 0.28 & 2.559 & Neutral & Benign & \\
\hline Ile462Met & Tolerated & 0.16 & 0.233 & Neutral & Benign & \\
\hline
\end{tabular}


Safder I et al. (2021). Not Bot Horti Agrobo 49(3):12346

\begin{tabular}{|c|c|c|c|c|c|c|}
\hline Asp491Glu & Tolerated & 1 & 0.511 & Neutral & Benign & \\
\hline Val492Met & Affected & 0 & -2.467 & Neutral & Probably damaging & 0.973 \\
\hline Met500Ile & Tolerated & 0.73 & 1.228 & Neutral & Benign & \\
\hline Val526Leu & Tolerated & 0.46 & -1.888 & Neutral & Benign & \\
\hline Asn533Lys & Tolerated & 1 & 0.533 & Neutral & Benign & \\
\hline Ala536Ser & Affected & 0 & -0.279 & Neutral & Benign & \\
\hline Ala44Gly & Tolerated & 0.38 & -0.537 & Neutral & Benign & \\
\hline Thr49Ala & Tolerated & 0.1 & -0.571 & Neutral & Benign & \\
\hline Val50Gly & Tolerated & 0.07 & -0.242 & Neutral & Benign & \\
\hline Met52Thr & Tolerated & 0.05 & -2.242 & Neutral & Benign & \\
\hline Val95Leu & Tolerated & 0.64 & 0.973 & Neutral & Benign & \\
\hline Gly101Ala & Tolerated & 0.51 & 0.101 & Neutral & Benign & \\
\hline Gly103Ala & Tolerated & 0.59 & -0.942 & Neutral & Benign & \\
\hline Thr130Ala & Tolerated & 1 & 2.286 & Neutral & Benign & \\
\hline Ala167Thr & Tolerated & 0.32 & 0.039 & Neutral & Possibly damaging & 0.691 \\
\hline Tyr182Phe & Tolerated & 0.25 & -3.812 & Deleterious & Probably damaging & 1 \\
\hline Val234Ile & Tolerated & 0.59 & -0.048 & Neutral & Benign & \\
\hline Pro268Ser & Tolerated & 0.52 & -0.526 & Neutral & Possibly damaging & 0.859 \\
\hline Val549Ile & Affected & 0 & -0.659 & Neutral & Probably damaging & 1 \\
\hline
\end{tabular}

\section{Structural and functional modifications}

We submitted all the high risk nsSNPs to MuPred, to explore functional changes due to amino acid variations (Table 3). Deleterious nsSNPs influenced several mechanisms; some with high probability score in Arg355Pro (altered trans membrane protein), Pro157Leu (gain of helix), E62 (gain of Acetylation, loss of loop). Variations in V492M and V549I affected most mechanisms. Some amino acid variations had low probability score; therefore, no mechanism was detected. These functional changes revealed nsSNPs could influence functional variation in GPAT proteins.

Table 3. Effect of high-risk nsSNPs to rice GPATs functional and structural mechanisms

\begin{tabular}{|c|c|c|}
\hline Mutation & $\begin{array}{c}\text { Probability of deleterious } \\
\text { mutation }\end{array}$ & Molecular mechanism altered by mutations \\
\hline $\mathrm{R} 430 \mathrm{H}$ & 0.148 & \\
\hline $\mathrm{D} 36 \mathrm{H}$ & 0.185 & \\
\hline $\mathrm{E} 62 \mathrm{~K}$ & 0.627 & Gain of acetylation at E62 (0.0013) \\
\hline $\mathrm{A} 32 \mathrm{~V}$ & 0.084 & \\
\hline R355P & 0.925 & Altered transmembrane protein $(0.0086)$ \\
\hline A502P & 0.224 & \\
\hline P157L & 0.941 & Gain of Helix (0.02) \\
\hline $\mathrm{D} 36 \mathrm{H}$ & 0.185 & \\
\hline G447S & 0.456 & \\
\hline F128L & 0.761 & Altered Metal binding $(0.04)$ \\
\hline G289C & 0.655 & $\begin{array}{l}\text { Loss of Ubiquitylation at K284(0.03) } \\
\text { Altered Transmembrane protein }(0.03) \\
\text { Gain of Disulfide linkage at G289 (0.05) }\end{array}$ \\
\hline V492M & 0.66 & Altered DNA binding $(0.05)$ \\
\hline
\end{tabular}




\begin{tabular}{|c|c|c|}
\hline & & Altered DNA binding $(0.05)$ \\
& \multirow{4}{*}{0.542} & Gain of Relative solvent accessibility $(0.01)$ \\
& Altered Metal binding $(0.00026)$ \\
& Altered Disordered interface $(0.04)$ \\
& Altered Ordered interface $(0.05)$ \\
& Altered DNA binding $(0.0008)$ \\
& Gain of Allosteric site at N545(0.01) \\
\hline
\end{tabular}

\section{Effects on protein stability}

We used I-Mutant to analyze stability changes to GPAT proteins by the nsSNPs. This tool predicts the reliability index (RI) and energy change values. The results revealed that all the variations decreased the stability of GPAT proteins (Table 4). Protein stability is vital to maintain its three dimensional structure; a reduction in stability causes protein denaturation, unfolding, and protein aggregation.(Ortbauer et al., 2013; Deller et al., 2016).

Table 4. nsSNPs impact to protein stability, TM-Score and, RMSD values

\begin{tabular}{|c|c|c|c|c|c|c|c|}
\hline Gene ID & $\begin{array}{c}\text { nsSNP } \\
\text { position }\end{array}$ & Amino acid change & Stability & RI & DDG & TM-score & RMSD \\
\hline OsGPAT1 & 8350403 & R430H & Decrease & 9 & -1.12 & 0.51851 & 5.67 \\
\hline OsGPAT2 & 10972698 & E62K & Decrease & & & 0.49351 & 2.35 \\
\hline OsGPAT7 & 40868136 & A32V & Decrease & 6 & -0.67 & 0.52881 & 4.28 \\
\hline OsGPAT 8 & 776197 & R355P & Decrease & 0 & -0.32 & 0.42009 & 3.55 \\
\hline OsGPAT 16 & 22484156 & A502P & Decrease & 7 & -1.07 & 0.49196 & 4.48 \\
\hline OsGPAT17 & 24725803 & P157L & Decrease & 4 & -0.42 & 0.68016 & 3.8 \\
\hline OsGPAT 18 & 30123014 & D36H & Decrease & 5 & -1.09 & 0.46905 & 3.02 \\
\hline OsGPAT20 & 1757860 & G447S & Decrease & 4 & -0.96 & 0.48605 & 5.13 \\
\hline OsGPAT20 & 1758815 & F128L & Decrease & 6 & -0.85 & 0.58509 & 4.77 \\
\hline OsGPAT24 & 25201089 & G289C & Decrease & 7 & -0.92 & 0.783 & 2.88 \\
\hline OsGPAT25 & 27489854 & V492M & Decrease & 7 & -0.86 & 0.4237 & 2.99 \\
\hline OsGPAT26 & 23092180 & Y182F & Decrease & 0 & -0.17 & 0.45534 & 3.85 \\
\hline OsGPAT26 & 23094109 & V549I & Decrease & 2 & 0.62 & 0.45009 & 3.31 \\
\hline
\end{tabular}

Free Energy change value (DDG); Reliability index (RI); Tm-Align score -similarity between native and mutant protein 3D models; Root mean square difference (RMSD)-variation between wild type and mutant protein; 3D model score

\section{Evolutionary conservation of amino acid residue positions in rice GPAT genes}

We used Consurf web tool to infer the evolutionary conservation of amino acid residues positions effected by nsSNPs. Consurf analyzes amino acid residues and allocate a conservation scale ranging between highly conserved, average, and variable to each amino acid residues, where variable reflect the lowest score. The score is calculated by merging the solvent accessibility predictions and evolutionary conservation data.

Table 5 demonstrates the conservation score of some high-risk nsSNPs in each GPATgene. We found that amino acid positions R355P, P157L, F128L, V549I were highly conserved, whereas R430H, E62K, A502P, D36H were conserved. These results suggested amino acid residues affected by nsSNPs are located at evolutionary conserved positions. We have shown two highly conserved and exposed nsSNPs structures in Figures $2 \mathrm{~A}$ and $2 \mathrm{~B}$ respectively.

Amino acid sites that are largely conserved, participates in important biological functions (Berezin et al., 2004; Arshad and Attya Bhatti, 2018).In this context, nsSNP positions which are also found in conserved positions are significant for protein functions. 
Table 5. Evolutionary conservation profile of amino acid positions affected by high-risk nsSNPs

\begin{tabular}{|c|c|c|c|c|}
\hline Gene ID & nsSNP position & Amino acid change & Conservation score & Prediction \\
\hline OsGPAT1 & 8350709 & $\mathrm{R} 430 \mathrm{H}$ & 6 & Conserved (e) \\
\hline OsGPAT2 & 10972698 & E62K & 7 & Conserved (e) \\
\hline OsGPAT7 & 40868136 & $\mathrm{~A} 32 \mathrm{~V}$ & 8 & buried residue $(\mathrm{b})$ \\
\hline OsGPAT\& & 776197 & $\mathrm{R} 355 \mathrm{P}$ & 9 & $\begin{array}{l}\text { Highly conserved and exposed } \\
\text { (f) }\end{array}$ \\
\hline OsGPAT 16 & 22484156 & A $502 \mathrm{P}$ & 2 & Conserved $(\mathrm{e})$ \\
\hline OsGPAT 17 & 24725803 & P157L & 9 & highly conserved and buried (s) \\
\hline OsGPAT 18 & 30123014 & $\mathrm{D} 36 \mathrm{H}$ & 2 & Conserved $(\mathrm{e})$ \\
\hline OsGPAT20 & 1757860 & G447S & 5 & exposed residue $(\mathrm{e})$ \\
\hline OsGPAT 20 & 1758815 & F128L & 9 & $\begin{array}{l}\text { Highly conserved and exposed } \\
\text { (f) }\end{array}$ \\
\hline OsGPAT24 & 25201089 & G289C & 5 & exposed residue $(\mathrm{e})$ \\
\hline OsGPAT 25 & 27487401 & $\mathrm{D} 146 \mathrm{~N}$ & 6 & exposed residue (e) \\
\hline OsGPAT 26 & 23092180 & $\mathrm{Y} 182 \mathrm{~F}$ & 6 & buried residue $(\mathrm{b})$ \\
\hline OsGPAT 26 & 23094109 & V549I & 8 & $\begin{array}{l}\text { Highly conserved and exposed } \\
\text { (f) }\end{array}$ \\
\hline
\end{tabular}

The Conservation scale:

Variable: 1-3, Average: 4-6, Conserved amino acid: 7-9; (f): highly conserved and exposed; (s): highly conserved and buried (e): exposed amino acid residues, (b): buried amino acid residue

\section{Structural analysis and modeling of wild type and mutant protein $3 D$ structures}

To explore whether the high risk nsSNPs impact protein structure, we generated 3D protein models of native and variant structures in genes carrying high impact nsSNPs mentioned in Table 4. We initially used Phyre2 that generated structures in pdb format, and then visualized by using Chimera. Besides, we analyzed the TM-align score and RMSD scores for the protein models (Table 4). A higher RMSD value demonstrates the deviation between mutant and the native model. Based on RMSD values, most mutant models showed high variation in structures with 2 or more RMSD value. Similarly, no model revealed low or zero value, demonstrating each nsSNPs have affected protein structures in some capacity. Finally, to demonstrate 3D structures, we selected four proteins having at least 2 RMSD values and low TM values. Figure 3 display the location of amino acid substitutions in each protein native and variant model. In these models, there were residual changes, along with variation in parameters including total energy, decrease in protein stability, suggesting they can influence protein folding. We further superimposed these variant models in figure 3 over wild types to explore the structural difference between them. Every superimposed model showed a difference in 3D structure, suggesting these nsSNPs affected the protein structure (Figure 4). 


\section{Conservation profile of Amino acids in OsGPAT 17}

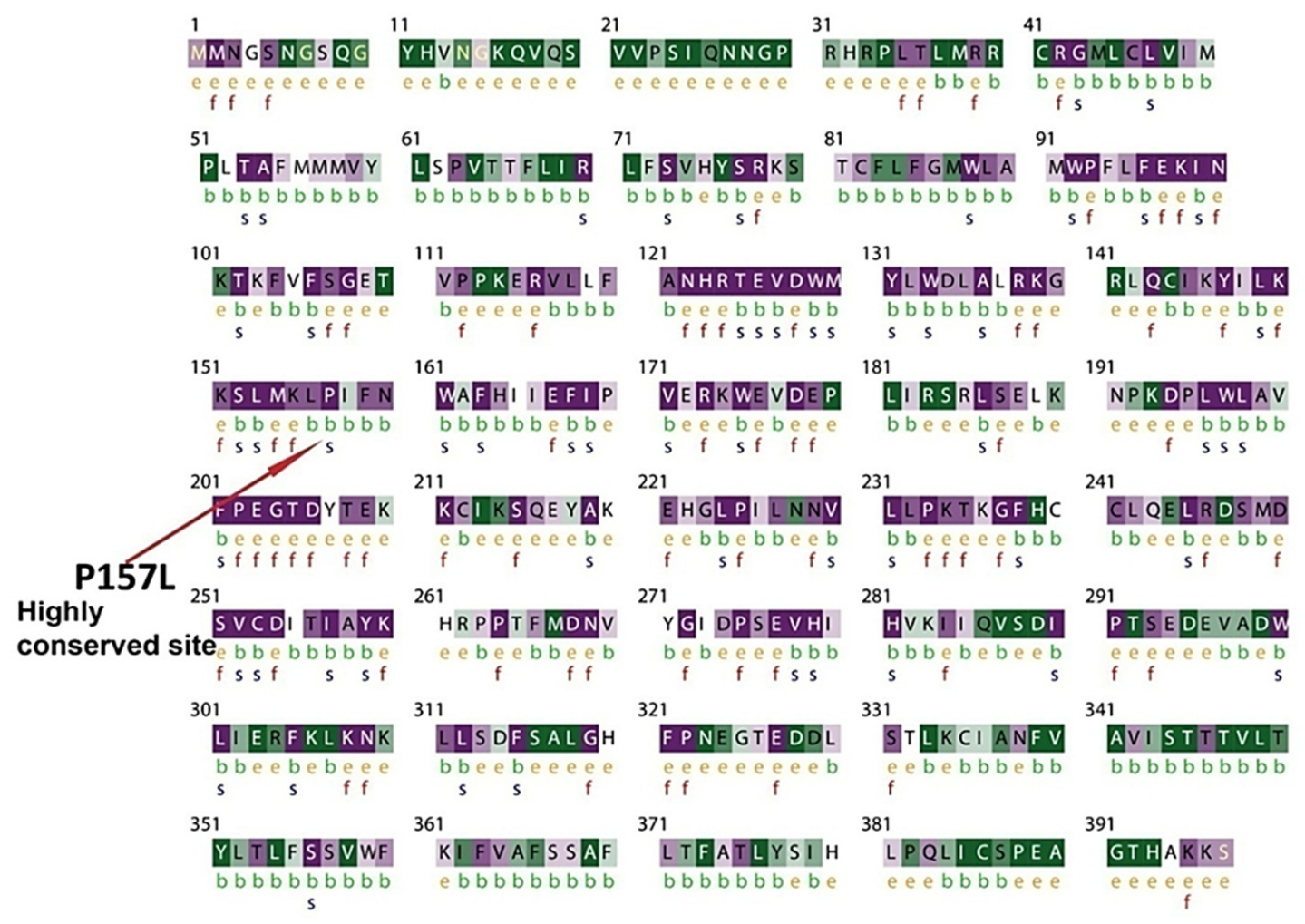

The conservation scale:

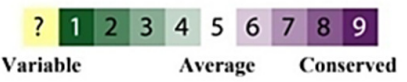

e - An exposed residue according to the neural-network algorithm.

b - A buried residue according to the neural-network algorithm.

$f$ - A predicted functional residue (highly conserved and exposed).

$s$ - A predicted structural residue (highly conserved and buried).

- Insufficient data - the calculation for this site was

performed on less than $10 \%$ of the sequences.

Figure 2A. Evolutionary conservation profile in OsGPAT 17 demonstrating P 157 amino acid position Amino acid residues conservation profile demonstrating the extent of evolutionary conservation. The conservation scale ranged from 1-10, where 10 is the highly conserved and 1 the lowest. Protein regions associated with function tend to be highly conserved over the course of evolution 


\section{Conserved Amino Acid profile in OsGPAT 8}

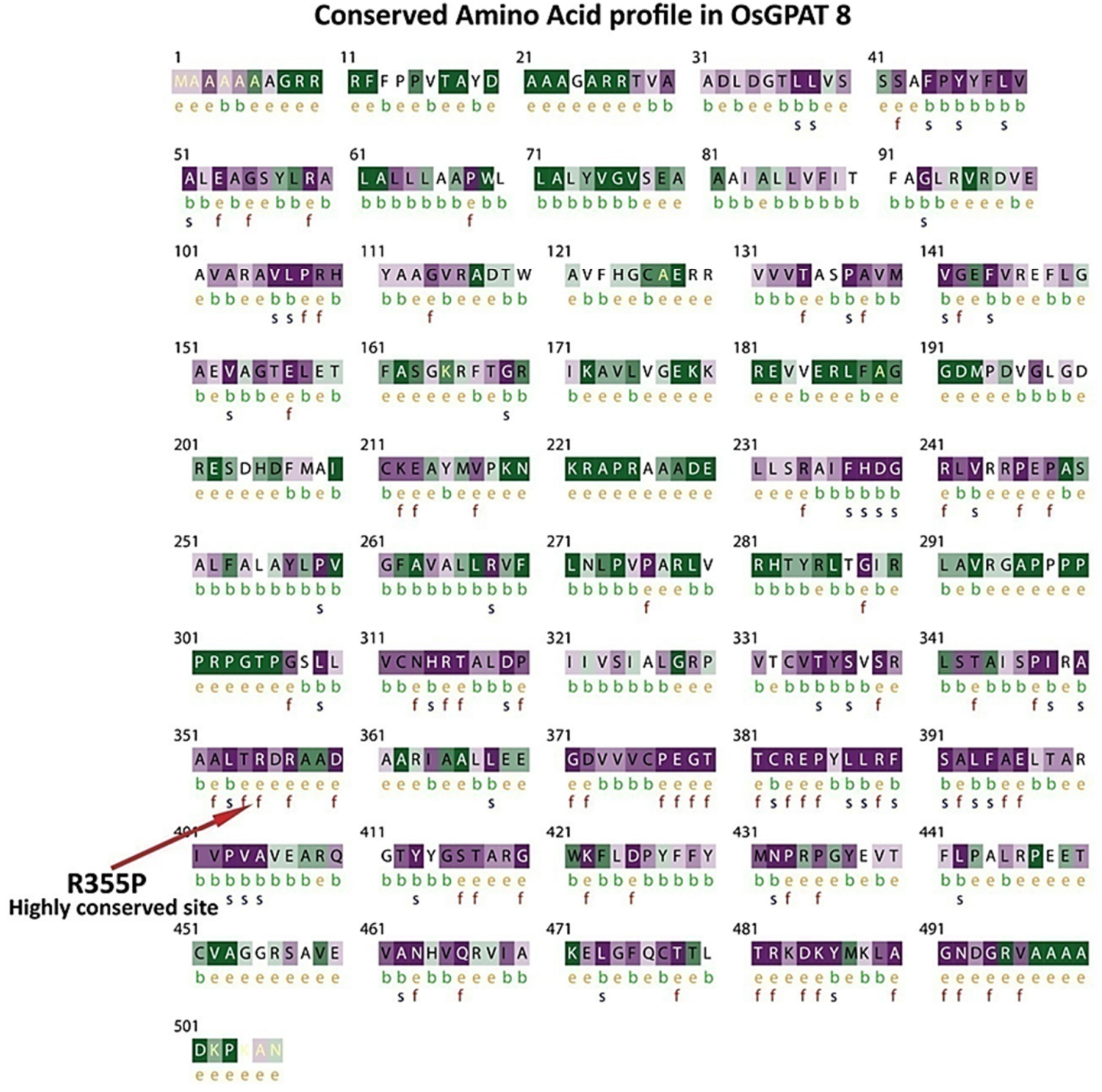

Figure 2B. Evolutionary conservation profile in OsGPAT 8 demonstrating R355P amino acid position Amino acid residues conservation profile demonstrating the extent of evolutionary conservation. The conservation scale ranged from 1-10, where 10 is the highly conserved and 1 the lowest. Protein regions associated with function tend to be highly conserved over the course of evolution 

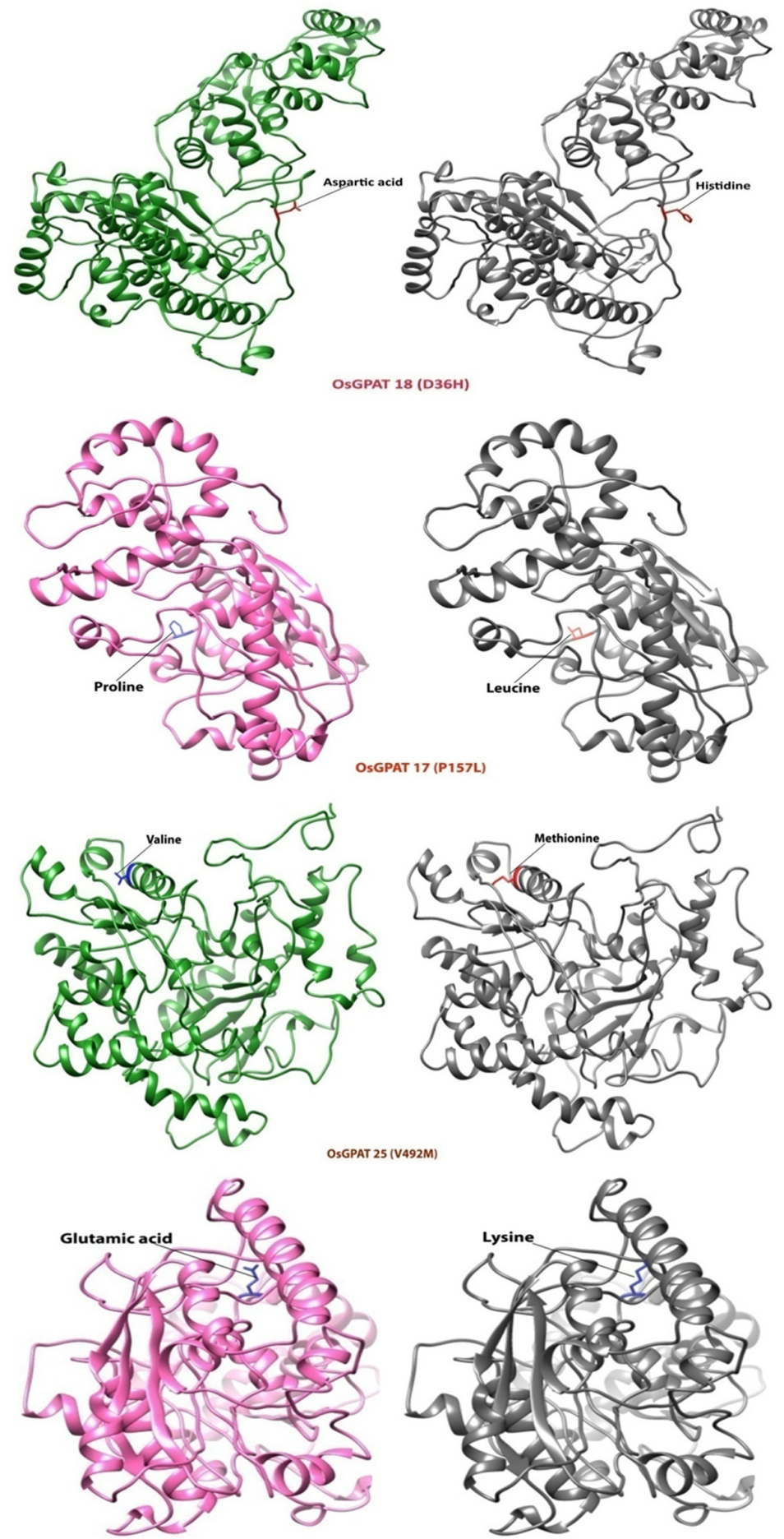

Figure 3. Protein 3D models of the rice GPAT proteins at native and variant positions generated by Phyre Full-length models of GPAT proteins monomer having native residues on left sides and variant residues on the right side. Arrows indicate the specific location of substitution at amino acid residues derived from important SNPs. Every picture represents the substituted amino acid and the residual change in the protein structure. Each amino acid residue is demonstrated with color 

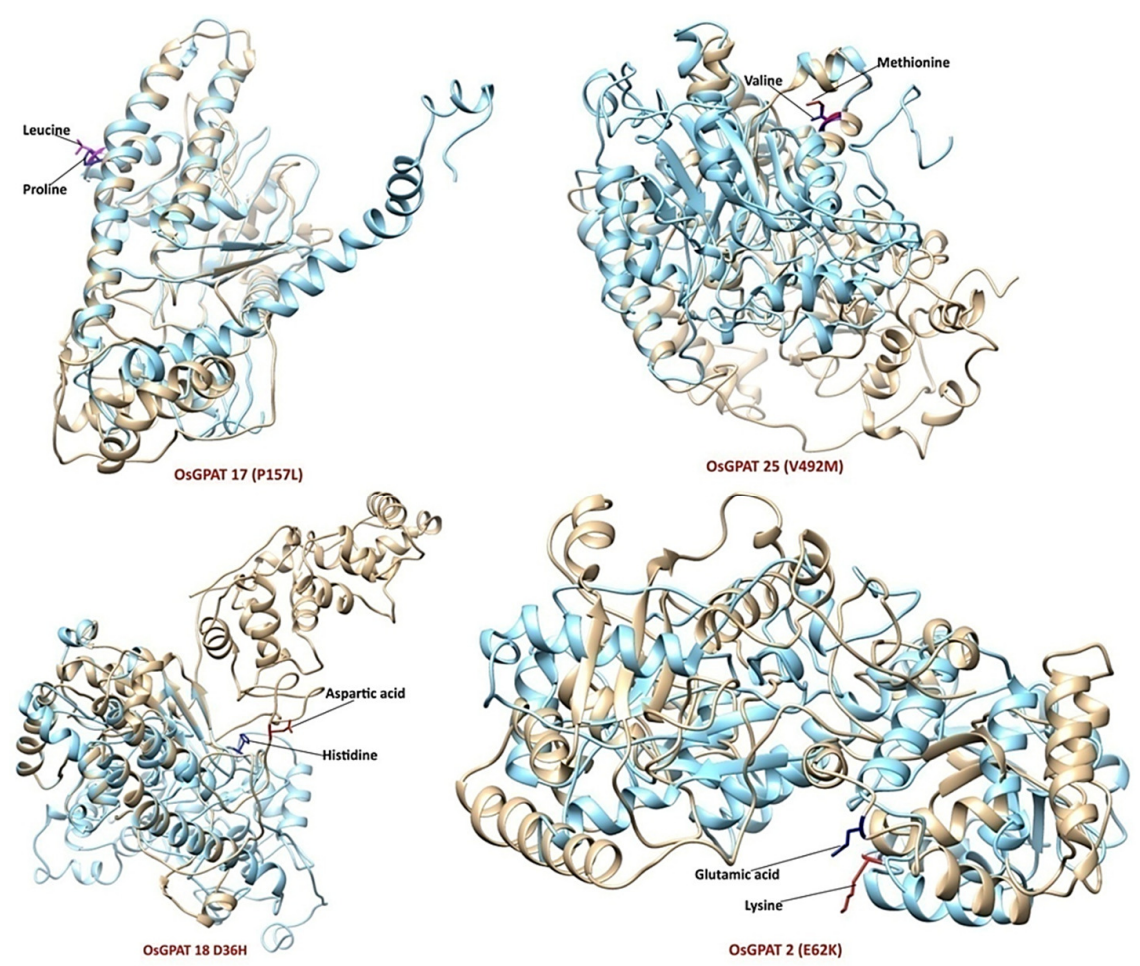

Figure 4. Superimposed 3D structures of selected native and variant rice GPAT proteins caused by the high-risk nsSNPS

Superimposed models were created by Chimera software. The arrows demonstrate the variant positions in superimposed protein models. Native and variant models are shown by blue and brown colors respectively

\section{Post Translational modification prediction}

Post translational modifications (PTM) influence protein conformation, stability, activity, localization and their interactions. They provide proteome diversity, impact signaling pathways, influence gene expression and enzyme kinetics (Piquerez et al., 2014; Friso and van Wijk, 2015). Therefore, we estimated if nsSNPS resided on the PTM sites and they had any influence on PTM sites. We utilized many in silico tools to predict PTM sites in GPAT proteins. We used at least two in silico tools for each PTM event including the phosphorylation, methylation and ubiquitylation and selected positions predicted by both tools. We also explored whether any PTM site was conserved as well.

We investigated Phosphorylation sites by GPS 5.0 and NetPhos 3.1; both tools predicted 100 sites in various rice GPAT proteins (Table S2). 79 amino acid residues were Serine, 16 residues were Threonine and only 4 residues were Tyrosine. For methylation PTMs of lysine residues PSSMe and iMethyl-PseAAC tools were used. We considered those sites predicted by both these tools at 0.5 SVM Probability thresholds. There were 127 lysine residues that were common in both tools (Table S3). We used BDM-PUB and Ub-Pred to predict Ubiquitylation sites. 36 common amino acid sites were predicted for having a potential ubiquitylation site (Table S4).

We used Consurf results to explore the highly conserved PTM sites (Table S5). But we could not find any nsSNP coincided with the conserved PTM sites. Past studies have demonstrated a variation in the conserved PTM site affect protein function. 


\section{Discussion}

The current study demonstrates various nonsynonymous SNPs could disturb rice GPAT protein structure and function. We filtered the nsSNPs (Table 1), shortlisted the high risk or deleterious SNPs (Table 2 ), and analysed their impact on protein structure and functions.

Initially, we find 1056 SNPs among 100 Chinese rice varieties in 26 GPATgenes (Table S6) as a model gene family, we identified in a genome-wide study (Safder et al., 2021). We shortlisted 98 coding nsSNPs from these $1056 \mathrm{SNPs}$, that can change amino acid residues and alter protein function. We further used various computational tools including Provean, SIFT, Polyphen-2 to find significant non-synonymous SNPs having deleterious or potentially damaging effects to proteins. Since every computational tool uses different algorithms, their output could slightly differ; still, we find similarity in the predictions. For instance, many nsSNPs predicted as deleterious by an individual tool (Table 2), were often predicted by a second tool. Though these nsSNPs (predicted by a single tool in Table 2) can't be neglected and should be considered important, we only selected nsSNPs predicted by all the tools to strengthen our results. Hence, we found $13 \mathrm{SNPs}$ (predicted by all three tools), considered as high-risk nsSNPs among the $98 \mathrm{nsSNPs}$ (Table 3). We also explored functional PTM amino acid residues at phosphorylation, ubiquitylation and methylation sites, and investigated if the nsSNPs coincide with the PTM site; as modification in PTM sites can alter protein function.

SNPs can impact phenotypic diversity among different plant traits, influence gene expression, and are associated with various functions (Kharabian-Masouleh et al., 2012; Wang et al., 2018; Wang et al., 2020b). Therefore, SNP identification and their functional elucidation provide a better understanding of their effects on gene functions and their phenotypes. Identifying functional SNPs is a complicated task in large population studies. Hence, it is beneficial to prior explore putative functional SNPs.

Past studies suggest nsSNPs influence different mechanisms. Biosynthesis of secondary metabolite, and signal transduction pathways are involved in fruit ripening and defense responses. A change in the cell wall structure and starch conversion to sugar, play roles in fruit ripening. Deleterious nsSNPs are involved in these signal transduction and metabolic pathways. An SNP in tomato invertase gene changed amino acid residue near proteins catalytic site and affected enzyme activity. In rice, 66 functional SNPs were discovered from 18 genes involved in starch biosynthesis. An important SNP was reported at the 1188 nucleotide position in Glucose-6-Phosphate Translocator 1 (GPT1), changed amino acid residues associated with amylase content. In another study, SNPs in several genes were identified involving in maize root development and associated with seedling root traits. Four functional SNPs in the HSP17.8 gene in barley varieties were associated with agronomic traits. Similarly, in tomato, functional SNPs affect gene expression and are associated with phenotypic differences among the tomato lines. These examples demonstrate functional SNPs influence gene functions, reflecting the significance of the current study, as the identified nsSNPs could have a major effect on GPAT genes (Kharabian-Masouleh et al., 2012; Hirakawa et al., 2013; Seymour et al., 2013; Xia et al., 2013; Kumar et al., 2014; Schreiber et al., 2014; Bhardwaj et al., 2016; Huq et al., 2016; Zaynab et al., 2018).

In past studies we find, in-silico approaches used in humans, plants, and identified functionally significant SNPs (De Alencar and Lopes, 2010; Kharabian, 2010; Kamaraj and Purohit, 2013; Arshad and Attya Bhatti, 2018; Zhang et al., 2020). In rice, the Granule Bound Starch Synthase I GBSSI was used as a model plant gene and explored functional SNPs. The study identified a candidate SNP imparting a major impact on GBSSI and its phenotype. This nsSNP at exon 6, showed the highest effect on amylose content according to the SIFT prediction results (Kharabian, 2010), also reported in a previous study (Chen et al., 2008), suggesting coherence between in silico analysis results and experimental approaches. Although experimental approaches provide strong evidence for estimating SNPs functional significance, they are tedious, lack exactness in phenotypic and genotypic data, so these experiments are not easy to detect SNPs (Cobb et al., 2013). Besides, high false positive results crop up due to population structures and multiple testing. These

results, therefore, mislead when a casual SNP is considered more significant in contrast to a truly casual variant (Tibbs Cortes et al.). 
Computational methods are used to explore functional variants to reduce the burden from statistical association studies. Therefore, in silico tools provide an opportunity to facilitate experimental approaches and they can be used to recruit, identify and characterize functionally important SNPs having a major impact on phenotype (Tam et al., 2019). In this regard, an in-silico study identified SNP diversity in cultivated and wild tomato genomes, investigated 1838 nsSNPs among 988 genes. There were 28 deleterious SNPs distributed among 27 genes predicted in hormonal and plant pathogen pathways similar to the current study. Further, they selected nsSNPs deleterious effect on the protein structure (Bhardwaj et al., 2016). Likewise, a study explored important SNPs in the TGF- $\beta$ receptor type 3 gene in chicken by using tools Sift, PANTHER, and I-mutant, and found a nsSNP in the coding region with deleterious impact, decreasing the protein stability (Rasal et al., 2015). Another study predicted putative effects of SNPs on 58 Prunus rootstocks genes using SnpEff. The SNPs were categorized as a modifier, low, moderate, and high impact; high impact SNPs were further explored using in silico tools (Guajardo et al., 2020).

Our results identified many high impact nsSNPs found in GPATs conserved protein regions. For instance, nsSNPs including P157L, R355P, F128L, V549I, and A32V located at highly conserved positions (Table 5). Past studies have suggested mutations in conserved regions can affect protein functions. Mutations in the ZP domain in the TGFBR3 gene affected its protein function; a conserved amino acid is essential for product and substrate specificity in triterpene synthases. Regarding variation between native and variant protein structures, we found most protein models had high RMSD value (two or more), reflecting differences between native and variant models affecting the protein stability (Jovine et al., 2002; Han et al., 2006; Salmon et al., 2016; Islam et al., 2019; Liao et al., 2019; Bhardwaj and Purohit, 2020).

We could not find any PTM site coincided with the nsSNP, but many PTM sites were highly conserved. Past studies demonstrated PTM sites influence protein function, if there is a variation in their conserved positions, and affect protein stability or inter protein structures (Arif et al., 2017; Gulzar et al., 2017).

\section{Conclusions}

Briefly, in the study we identified all candidate SNPs most likely to affect rice GPAT proteins and influence their function. We demonstrated in silico tools could help us to characterize functional SNPs which possibly have potential impact on GPAT genes and related phenotypes. These functional SNPs could provide value in developing functional markers by associating their link to phenotypic traits. The study will also provide a computational pathway to find candidate SNPs in other rice gene families.

\section{Authors' Contributions}

Conceptualization, I.S., G.S., P.H. and S.T.; Data curation, I.S., G.S., P.H. and S.T.; Formal analysis, I.S., G.S., Z.S.; Funding acquisition, P.H. and S.T.; Methodology, I.S., G.S., Z.S.; Project administration, P.H. and S.T.; Resources, P.H. and S.T.; Supervision, P.H. and S.T.; Validation, Z.S.; Writing - original draft, I.S. and G.S.; Writing - review \& editing, P.H. and S.T. All authors read and approved the final manuscript. 


\section{Acknowledgements}

We appreciate the support of the Central Public-interest Scientific Institution Basal Research Fund of China (No. Y2020PT07) and the Chinese Academy of Agricultural Sciences (CAAS) Agricultural Sciences and Technologies Innovation Program for the financial assistance. We are also thankful to the China Scholarship Council (CSC) for providing the fellowship to Mr. Imran Safder (CSC No: 2017SLJ022683) during this research project.

\section{Conflict of Interests}

The authors declare that there are no conflicts of interest related to this article.

\section{References}

Adzhubei IA, Schmidt S, Peshkin L, Ramensky VE, Gerasimova A, Bork P, ... Sunyaev SR (2010). A method and server for predicting damaging missense mutations. Nature Methods 7:248-249. https://doi.org/10.1038/nmeth0410-248

Alexandrov N, Tai S, Wang W, Mansueto L, Palis K, Fuentes RR, ... Li Z (2015). SNP-Seek database of SNPs derived from 3000 rice genomes. Nucleic Acids Research 43:D1023-D1027. https://doi.org/10.1093/nar/gku1039

Arif R, Akram F, Jamil T, Mukhtar H, Lee SF, Saleem M (2017). Genetic variation and its reflection on posttranslational modifications in frequency clock and mating Type a-1 proteins in Sordaria fimicola. BioMed Research International 2017:1268623. https://doi.org/10.1155/2017/1268623

Arshad M, Attya Bhatti PJ (2018). Identification and in silico analysis of functional SNPs of human TAGAP protein: A comprehensive study. PloS One 13(1):e0188143. https://doi.org/10.1371/journal.pone.0188143

Ashkenazy H, Abadi S, Martz E, Chay O, Mayrose I, Pupko T, Ben-Tal N (2016). ConSurf 2016: an improved methodology to estimate and visualize evolutionary conservation in macromolecules. Nucleic Acids Research 44:W344-W350. https://doi.org/10.1093/nar/gkw408

Ashkenazy H, Erez E, Martz E, Pupko T, Ben-Tal N (2010). ConSurf 2010: calculating evolutionary conservation in sequence and structure of proteins and nucleic acids. Nucleic Acids Research 38:W529-W533. https://doi.org/10.1093/nar/gkq399

Berezin C, Glaser F, Rosenberg J, Paz I, Pupko T, Fariselli P, Casadio R, Ben-Tal N (2004). ConSeq: the identification of functionally and structurally important residues in protein sequences. Bioinformatics 20:1322-1324. https://doi.org/10.1093/bioinformatics/bth070

Bhardwaj A, Dhar YV, Asif MH, Bag SK (2016). In silico identification of SNP diversity in cultivated and wild tomato species: insight from molecular simulations. Scientific Reports 6:38715-38715. https://doi.org/10.1038/srep38715

Bhardwaj VK, Purohit R (2020). Structural changes induced by substitution of amino acid 129 in the coat protein of Cucumber mosaic virus. Genomics 112:3729-3738. https://doi.org/10.1016/j.ygeno.2020.04.023

Blom N, Gammeltoft S, Brunak S (1999). Sequence and structure-based prediction of eukaryotic protein phosphorylation sites. Journal of Molecular Biology 294:1351-1362. https://doi.org/10.1006/jmbi.1999.3310

Carugo O, Pongor S (2001). A normalized root-mean-spuare distance for comparing protein three-dimensional structures. Protein Science 10:1470-1473. https://doi.org/10.1110/ps.690101

Celniker G, Nimrod G, Ashkenazy H, Glaser F, Martz E, Mayrose I, Pupko T, Ben-Tal N (2013). ConSurf: using evolutionary data to raise testable hypotheses about protein function. Israel Journal of Chemistry 53:199206. https://doi.org/10.1002/ijch.201200096

Chaisan T, Van K, Kim MY, Kim KD, Choi B-S, Lee S-H (2012). In silico single nucleotide polymorphism discovery and application to marker-assisted selection in soybean. Molecular Breeding 29:221-233. https://doi.org/10.1007/s11032-010-9541-y 
Chen M-H, Bergman CJ, Pinson SRM, Fjellstrom RG (2008). Waxy gene haplotypes: Associations with pasting properties in an international rice germplasm collection. Journal of Cereal Science 48:781-788. https://doi.org/10.1016/j.jcs.2008.05.004

Choi Y, Chan AP (2015). PROVEAN web server: a tool to predict the functional effect of amino acid substitutions and indels. Bioinformatics (Oxford, England) 31:2745-2747. https://doi.org/10.1093/bioinformatics/btv195

Choi Y, Sims GE, Murphy S, Miller JR, Chan AP (2012). Predicting the functional effect of amino acid substitutions and indels. PloS One 7. https://doi.org/10.1371/journal.pone.0046688

Cobb JN, DeClerck G, Greenberg A, Clark R, McCouch S (2013). Next-generation phenotyping: requirements and strategies for enhancing our understanding of genotype-phenotype relationships and its relevance to crop improvement. Theoretical and Applied Genetics 126:867-887. https://doi.org/10.1007/s00122-013-2066-0

De Alencar S, Lopes JC (2010). A comprehensive in silico analysis of the functional and structural impact of SNPs in the IGF1R gene. BioMed Research International 715139. https://doi.org/10.1155/2010/715139

Deller MC, Kong L, Rupp B (2016). Protein stability: a crystallographer's perspective. Acta Crystallographica Section F: Structural Biology Communications 72:72-95. https://doi.org/10.1107/S2053230X15024619

Friso G, van Wijk KJ (2015). Posttranslational protein modifications in plant metabolism. Plant Physiology 169:14691487. https://doi.org/10.1107/S2053230X15024619

Gailing O, Vornam B, Leinemann L, Finkeldey R (2009). Genetic and genomic approaches to assess adaptive genetic variation in plants: forest trees as a model. Physiologia Plantarum 137:509-519. https://doi.org/10.1111/j.13993054.2009.01263.x

Goff SA, Ricke D, Lan T-H, Presting G, Wang R, Dunn M, Glazebrook J, Sessions A, Oeller P, Varma H (2002). A draft sequence of the rice genome (Oryza sativa L. ssp. japonica). Science 296:92-100. https://doi.org/10.1126/science.1068275

Guajardo V, Solís S, Almada R, Saski C, Gasic K, Moreno MÁ (2020). Genome-wide SNP identification in Prunus rootstocks germplasm collections using genotyping-by-sequencing: phylogenetic analysis, distribution of SNPs and prediction of their effect on gene function. Scientific Reports 10:1467-1467. https://doi.org/10.1038/s41598-020-58271-5

Gulzar N, Dingerdissen H, Yan C, Mazumder R (2017). Impact of nonsynonymous single-nucleotide variations on posttranslational modification sites in human proteins. Protein Bioinformatics, Springer, pp 159-190. https://doi.org/10.1007/978-1-4939-6783-4_8

Han JH, Kerrison N, Chothia C, Teichmann SA (2006). Divergence of interdomain geometry in two-domain proteins. Structure (London, England, 1993) 14:935-945. https://doi.org/10.1016/j.str.2006.01.016

Hirakawa H, Shirasawa K, Ohyama A, Fukuoka H, Aoki K, Rothan C, Sato S, Isobe S, Tabata S (2013). Genome-wide SNP genotyping to infer the effects on gene functions in tomato. DNA Research 20:221-233. https://doi.org/10.1093/dnares/dst005

Huq MA, Akter S, Nou IS, Kim HT, Jung YJ, Kang KK (2016). Identification of functional SNPs in genes and their effects on plant phenotypes. Journal of Plant Biotechnology 43:1-11. https://doi.org/10.5010/JPB.2016.43.1.1

Islam MJ, Khan AM, Parves MR, Hossain MN, Halim MA (2019). Prediction of deleterious non-synonymous SNPs of human STK11 gene by combining algorithms, molecular docking, and molecular dynamics simulation. Scientific Reports 9:16426. https://doi.org/10.1038/s41598-019-52308-0

Jackson SA (2016). Rice: the first crop genome. Rice 9:1-3. https://doi.org/10.1186/s12284-016-0087-4

Jiang D, Ye Q-1, Wang F-S, Cao L (2010). The mining of citrus EST-SNP and its application in cultivar discrimination. Agricultural Sciences in China 9:179-190. https://doi.org/10.1016/S1671-2927(09)60082-1

Jovine L, Qi H, Williams Z, Litscher E, Wassarman PM (2002). The ZP domain is a conserved module for polymerization of extracellular proteins. Nature Cell Biology 4:457-461. https://doi.org/10.1038/ncb802

Kamaraj B, Purohit R (2013). In silico screening and molecular dynamics simulation of disease-associated nsSNP in TYRP1 gene and its structural consequences in OCA3. BioMed Research International 2013:697051-697051. https://doi.org/10.1155/2013/697051

Kelley LA, Mezulis S, Yates CM, Wass MN, Sternberg MJ (2015). The Phyre2 web portal for protein modeling, prediction and analysis. Nature Protocols 10:845. https://doi.org/10.1107/S2053230X15024619

Kharabian-Masouleh A, Waters DLE, Reinke RF, Ward R, Henry RJ (2012). SNP in starch biosynthesis genes associated with nutritional and functional properties of rice. Scientific Reports 2:557. https://doi.org/10.1038/srep00557

Kharabian A (2010). An efficient computational method for screening functional SNPs in plants. Journal of Theoretical Biology 265:55-62. https://doi.org/10.1016/j.jtbi.2010.04.017 
Korani W, Clevenger JP, Chu Y, Ozias-Akins P (2019). Machine learning as an effective method for identifying true single nucleotide polymorphisms in polyploid plants. Plant Genome 12. https://doi.org/10.3835/plantgenome2018.05.0023

Kumar B, Abdel-Ghani AH, Pace J, Reyes-Matamoros J, Hochholdinger F, Lübberstedt T (2014). Association analysis of single nucleotide polymorphisms in candidate genes with root traits in maize (Zea mays L.) seedlings. Plant Science 224:9-19. https://doi.org/10.1007/s11103-015-0314-1

Lek M, Karczewski KJ, Minikel EV, Samocha KE, Banks E, Fennell T, ... MacArthur DG (2016). Analysis of proteincoding genetic variation in 60,706 humans. Nature 536:285-291. https://doi.org/10.1038/nature19057

Li B, Krishnan VG, Mort ME, Xin F, Kamati KK, Cooper DN, Mooney SD, Radivojac P (2009a). Automated inference of molecular mechanisms of disease from amino acid substitutions. Bioinformatics 25:2744-2750. https://doi.org/10.1093/bioinformatics/btp528

Li J-Y, Wang J, Zeigler RS (2014). The 3,000 rice genomes project: new opportunities and challenges for future rice research. Gigascience 3:2047-217X. https://doi.org/10.1186/2047-217X-3-8

Li X, Gao X, Ren J, Jin C, Xue Y (2009b). BDM-PUB: Computational prediction of protein ubiquitination sites with a Bayesian discriminant method. https://doi.org/10.2174/1389202919666191014091250

Liao M-l, Somero GN, Dong Y-W (2019). Comparing mutagenesis and simulations as tools for identifying functionally important sequence changes for protein thermal adaptation. Proceedings of the National Academy of Sciences 116:679-688. https://doi.org/10.1073/pnas.1817455116

Majeed S, Rana IA, Atif RM, Ali Z, Hinze L, Azhar MT (2019). Role of SNPs in determining QTLs for major traits in cotton. Journal of Cotton Research 2:5. https://doi.org/10.1186/s42397-019-0022-5

Mammadov J, Aggarwal R, Buyyarapu R, Kumpatla S (2012). SNP markers and their impact on plant breeding. International Journal of Plant Genomics 2012:728398. https://doi.org/10.1155/2012/728398

Mansueto L, Fuentes RR, Borja FN, Detras J, Abriol-Santos JM, Chebotarov D, ... Alexandrov N (2016). Rice SNP-seek database update: new SNPs, indels, and queries. Nucleic Acids Research 45:D1075-D1081. https://doi.org/10.1093/nar/gkw1135

McCouch SR, Zhao K, Wright M, Tung C-W, Ebana K, Thomson M, Reynolds A, Wang D, DeClerck G, Ali ML (2010). Development of genome-wide SNP assays for rice. Breeding Science 60:524535. https://doi.org/10.1270/jsbbs.60.524

Nelson MR, Marnellos G, Kammerer S, Hoyal CR, Shi MM, Cantor CR, Braun A (2004). Large-scale validation of single nucleotide polymorphisms in gene regions. Genome Research 14:1664-1668. https://doi.org/10.1101/gr.2421604

Ng PC, Henikoff S (2003). SIFT: Predicting amino acid changes that affect protein function. Nucleic Acids Research 31:3812-3814. https://doi.org/10.1093/nar/gkg509

Ortbauer M, Vahdati K, Leslie C (2013). Abiotic stress adaptation: protein folding stability and dynamics. Abiotic StressPlant Responses and Applications in Agriculture 1:3-25. https://doi.org/10.5772/53129

Pea G, Aung HH, Frascaroli E, Landi P, Pè ME (2013). Extensive genomic characterization of a set of near-isogenic lines for heterotic QTL in maize (Zea mays L.). BMC Genomics 14:61. https://doi.org/10.1186/1471-2164-14-61

Pettersen EF, Goddard TD, Huang CC, Couch GS, Greenblatt DM, Meng EC, Ferrin TE (2004). UCSF Chimera-A visualization system for exploratory research and analysis. Journal of Computational Chemistry 25:1605-1612. https://doi.org/10.1002/jcc.20084

Piquerez SJ, Balmuth AL, Sklenár J, Jones AM, Rathjen JP, Ntoukakis V (2014). Identification of post-translational modifications of plant protein complexes. JoVE Journal of Visualized Experiments e51095. https://doi.org/10.3791/51095

Qiu W-R, Xiao X, Lin W-Z, Chou K-C (2014). iMethyl-PseAAC: identification of protein methylation sites via a pseudo amino acid composition approach. BioMed Research International 947416. https://doi.org/10.1155/2014/947416

Radivojac P, Vacic V, Haynes C, Cocklin RR, Mohan A, Heyen JW, Goebl MG, Iakoucheva LM (2010). Identification, analysis, and prediction of protein ubiquitination sites. Proteins: Structure, Function, and Bioinformatics 78:365-380. https://doi.org/10.1002/prot.22555

Rasal KD, Shah TM, Vaidya M, Jakhesara SJ, Joshi CG (2015). Analysis of consequences of non-synonymous SNP in feed conversion ratio associated TGF- $\beta$ receptor type 3 gene in chicken. Meta Gene 4:107-117. https://doi.org/10.1016/j.mgene.2015.03.006 
Safder I, Shao G, Sheng Z, Hu P, Tang S (2021). Identification and analysis of the structure, expression and nucleotide polymorphism of the GPAT gene family in rice. Plant Gene 100290. https://doi.org/10.1016/j.plgene.2021.100290

Salmon M, Thimmappa RB, Minto RE, Melton RE, Hughes RK, O’Maille PE, Hemmings AM, Osbourn A (2016). A conserved amino acid residue critical for product and substrate specificity in plant triterpene synthases. Proceedings of the National Academy of Sciences 113:E4407-E4414. https://doi.org/10.1073/pnas.1605509113

Sandhu D, Pudussery MV, Kumar R, Pallete A, Markley P, Bridges WC, Sekhon RS (2020). Characterization of natural genetic variation identifies multiple genes involved in salt tolerance in maize. Functional \& Integrative Genomics 20:261-275. https://doi.org/10.1007/s10142-019-00707-X

Schreiber L, Nader-Nieto AC, Schönhals EM, Walkemeier B, Gebhardt C (2014). SNPs in genes functional in starchsugar interconversion associate with natural variation of tuber starch and sugar content of potato (Solanum tuberosum L.). G3 Genes, Genomes, Genetics 4:1797-1811. https://doi.org/10.1534/g3.114.012377

Senior AW, Evans R, Jumper J, Kirkpatrick J, Sifre L, Green T, ... Hassabis D (2020). Improved protein structure prediction using potentials from deep learning. Nature 577:706-710. https://doi.org/10.1038/s41586-0191923-7

Seymour GB, Chapman NH, Chew BL, Rose JK (2013). Regulation of ripening and opportunities for control in tomato and other fruits. Plant Biotechnology Journal 11:269-278. https://doi.org/10.1111/j.1467-7652.2012.00738.x

Tam V, Patel N, Turcotte M, Bossé Y, Paré G, Meyre D (2019). Benefits and limitations of genome-wide association studies. Nature Reviews Genetics 20:467-484. https://doi.org/10.1038/s41576-019-0127-1

Tibbs Cortes L, Zhang Z, Yu J (2021). Status and prospects of genome-wide association studies in plants. The Plant Genome 14:e20077._https://doi.org/10.1002/tpg2.20077

Wang C-C, Yu H, Huang J, Wang W-S, Faruquee M, Zhang F, ... Zheng T-Q (2020a). Towards a deeper haplotype mining of complex traits in rice with RFGB v2.0. Plant Biotechnology Journal 18:14-16. https://doi.org/10.1111/pbi.13215

Wang H, Ham T-H, Im D-E, Lar SM, Jang S-G, Lee J, Mo Y, Jeung J-U, Kim ST, Kwon S-W (2020b). A new SNP in rice gene encoding pyruvate phosphate dikinase (PPDK) associated with floury endosperm. Genes (Basel) 11:465. https://doi.org/10.3390/genes11040465

Wang H, Mo Y-J, Im D-E, Jang S-G, Ham T-H, Lee J, Jeung J-U, Kwon S-W (2018). A new SNP in cy OsPPDK gene is associated with floury endosperm in Suweon 542. Molecular Genetics and Genomics 293:1151-1158. https://doi.org/10.3390/genes11040465

Wen P-P, Shi S-P, Xu H-D, Wang L-N, Qiu J-D (2016). Accurate in silico prediction of species-specific methylation sites based on information gain feature optimization. Bioinformatics 32:3107-3115. https://doi.org/10.1093/bioinformatics/btw377

Withana WVE, Kularathna RMRE, Kottearachchi NS, Kekulandara DS, Weerasena J, Steele KA (2020). In silico analysis of the fragrance gene (badh2) in Asian rice (Oryza sativa L.) germplasm and validation of allele specific markers. Plant Genetic Resources: Characterization and Utilization 18:71-80. https://doi.org/10.1017/S1479262120000015

Xia Y, Li R, Ning Z, Bai G, Siddique KH, Yan G, Baum M, Varshney RK, Guo P (2013). Single nucleotide polymorphisms in HSP17. 8 and their association with agronomic traits in barley. PloS One 8:e56816. https://doi.org/10.1371/journal.pone.0056816

Xue Y, Zhou F, Zhu M, Ahmed K, Chen G, Yao X (2005). GPS: a comprehensive www server for phosphorylation sites prediction. Nucleic Acids Research 33:W184-W187. https://doi.org/10.1093/nar/gki393

Yang J, Anishchenko I, Park H, Peng Z, Ovchinnikov S, Baker D (2020). Improved protein structure prediction using predicted interresidue orientations. Proceedings of the National Academy of Sciences 117:1496-1503. https://doi.org/10.1073/pnas.1914677117

Yang W, Bai X, Kabelka E, Eaton C, Kamoun S, van der Knaap E, David F (2004). Discovery of single nucleotide polymorphisms in Lycopersicon esculentum by computer aided analysis of expressed sequence tags. Molecular Breeding 14:21-34. https://doi.org/10.1023/B:MOLB.0000037992.03731.a5

Zaynab M, Fatima M, Abbas S, Sharif Y, Umair M, Zafar MH, Bahadar K (2018). Role of secondary metabolites in plant defense against pathogens. Microbial 124:198-202. https://doi.org/10.1016/j.micpath.2018.08.034 
Zhang M, Huang C, Wang Z, Lv H, Li X (2020). In silico analysis of non-synonymous single nucleotide polymorphisms (nsSNPs) in the human GJA3 gene associated with congenital cataract. BMC Molecular and Cell Biology 21:12. https://doi.org/10.1186/s12860-020-00252-7

Zhang W, Mirlohi S, Li X, He Y (2018). Identification of functional single-nucleotide polymorphisms affecting leaf hair number in Brassica rapa. Plant Physiology 177:490-503. https://doi.org/10.1104/pp.18.00025

Zhang Y, Skolnick J (2005). TM-align: a protein structure alignment algorithm based on the TM-score. Nucleic Acids Research 33:2302-2309. https://doi.org/10.1093/nar/gki524

Zhao H, Yao W, Ouyang Y, Yang W, Wang G, Lian X, ... Xie W (2015). RiceVarMap: a comprehensive database of rice genomic variations. Nucleic Acids Research 43:D1018-D1022. https://doi.org/10.1093/nar/gku894
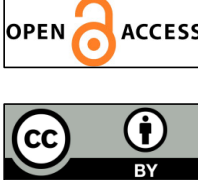

The journal offers free, immediate, and unrestricted access to peer-reviewed research and scholarly work. Users are allowed to read, download, copy, distribute, print, search, or link to the full texts of the articles, or use them for any other lawful purpose, without asking prior permission from the publisher or the author.

License - Articles published in Notulae Botanicae Horti Agrobotanici Cluj-Napoca are Open-Access, distributed under the terms and conditions of the Creative Commons Attribution (CC BY 4.0) License. (c) Articles by the authors; UASVM, Cluj-Napoca, Romania. The journal allows the author(s) to hold the copyright/to retain publishing rights without restriction. 
Safder I et al. (2021). Not Bot Horti Agrobo 49(3):12346

\section{Supplementary Files}

Table S1. 100 rice accessions from different regions of China using 3000 genome project data

\begin{tabular}{|c|c|c|}
\hline $\begin{array}{c}\text { Accession number/ } \\
\text { Sequence ID in 3KRG data }\end{array}$ & Variety name/Source & Indica/Japonica \\
\hline B060 & Aijiaonante & Indica \\
\hline B061 & Guangluai 4 & Indica \\
\hline B062 & Nantehao & Indica \\
\hline B064 & Xiangzaoxian 7 & Indica \\
\hline B067 & Funingzipigengzi & Indica \\
\hline B070 & Laoguangtou 83 & japonica \\
\hline B072 & Qiuqianbai & Indica \\
\hline B073 & Jinxibai2 & Indica \\
\hline B074 & Taishannuo & Indica \\
\hline B075 & Jinbaoyin & Indica \\
\hline B076 & Minbeiwanxian & Indica \\
\hline B079 & Esiniu & Indica \\
\hline B081 & Heidu 4 & Indica \\
\hline B082 & Qiyuexian & Indica \\
\hline B083 & Dongtingwanxian & Indica \\
\hline B101 & Yangkenuo & japonica \\
\hline B105 & Baoxuan 21 & Indica \\
\hline B106 & Wenxiangnuo & Indica \\
\hline B108 & Xianggu & Indica \\
\hline B112 & Liusha 1 & Indica \\
\hline B113 & Chenwan 3 & Indica \\
\hline B118 & Ergangai & Indica \\
\hline B119 & Guangluai 15 & Indica \\
\hline $\mathrm{B} 120$ & Hongwan 1 & Indica \\
\hline B123 & Zaoshuxiangheimi & Indica \\
\hline B124 & Geng 87-304 & japonica \\
\hline B126 & Zaoxian 240 & Indica \\
\hline B141 & Geng 7623 & Indica \\
\hline B142 & Ninghui 21 & japonica \\
\hline B143 & $76-1$ & japonica \\
\hline B147 & Baikehanhe & Indica \\
\hline B149 & Haoxiang & Indica \\
\hline B151 & Jinnante 43B & Indica \\
\hline B152 & Zaoshunonghu 6 & japonica \\
\hline B158 & Taizhong 65 & japonica \\
\hline B162 & Baigedao & japonica \\
\hline B163 & Liushizao & Indica \\
\hline B164 & Qingke & Aus \\
\hline B165 & Haohuangla & Indica \\
\hline B196 & Taidongludao & japonica \\
\hline B197 & Taizhongxianxuan 2 & Indica \\
\hline B198 & Jiefangxian & Indica \\
\hline B199 & Hongmisandan & japonica \\
\hline B200 & Jinyou 1 & Indica \\
\hline B202 & Baoxie 123B & Indica \\
\hline B203 & Biwusheng & Indica \\
\hline B204 & Longhuamaohu & japonica \\
\hline B205 & Cunsanli & japonica \\
\hline B207 & Aihechi & Indica \\
\hline B222 & Beizinuo & Indica \\
\hline B223 & Cungunuo & japonica \\
\hline B224 & Younian & Indica \\
\hline B225 & Guantuibaihe 1 & japonica \\
\hline B226 & Heimangdao & japonica \\
\hline B227 & Menjiagao 1 & Indica \\
\hline
\end{tabular}




\begin{tabular}{|c|c|c|}
\hline B228 & Haobayong 1 & japonica \\
\hline B229 & Menjiading 2 & Indica \\
\hline $\mathrm{B} 230$ & Banjiemang2 & japonica \\
\hline $\mathrm{B} 232$ & Xiangaizao 10hao & Indica \\
\hline $\mathrm{B} 233$ & Xiangwanxian 1 & Indica \\
\hline B235 & Zhonghua 8 & Indica \\
\hline $\mathrm{B} 236$ & Jindao 1 & Indica \\
\hline $\mathrm{B} 238$ & Momi & Indica \\
\hline B239 & Zhendao 232 & Indica \\
\hline B240 & Zhengdao 5 & japonica \\
\hline B241 & Lamujia & japonica \\
\hline B242 & Gui 630 & Indica \\
\hline B243 & Huhui 91269 & Aus \\
\hline B244 & Xiangdao & Indica \\
\hline B246 & Laozaogu & Indica \\
\hline B247 & Jinnante B & Indica \\
\hline B248 & Zhuzhen B & Indica \\
\hline B249 & Chaoyangyihao B & Indica \\
\hline B250 & Annongwangeng B & japonica \\
\hline $\mathrm{B} 253$ & Jiangnongzao $1 \mathrm{~B}$ & Indica \\
\hline B252 & Xiangai B & Indica \\
\hline IRIS_313-10459 & Pi 160862-1 & japonica \\
\hline IRIS_313-10562 & Seng-Chui-Lin & japonica \\
\hline IRIS_313-11654 & PL 3165 & Indica \\
\hline IRIS_313-11664 & Cun Gu Nuo & Indica \\
\hline IRIS_313-11665 & Jin Hua 258 & Indica \\
\hline IRIS_313-11666 & Long Ge 33 & Indica \\
\hline IRIS_313-11667 & Luo Ai Zao 3 & Indica \\
\hline IRIS_313-11668 & Rong Dao 4 & Indica \\
\hline IRIS_313-11669 & $\mathrm{F} 478$ & Indica \\
\hline IRIS_313-11726 & Guang Qing 334 & Indica \\
\hline IRIS_313-11727 & Hong Yang Zao 3 & Indica \\
\hline IRIS_313-11728 & Luo Si Zhan & Indica \\
\hline IRIS_313-11729 & Mei Liu Zao 5 & Indica \\
\hline IRIS_313-11730 & Qing Er Xiao 2 & Indica \\
\hline IRIS_313-11731 & Qing Tai Ai & Indica \\
\hline IRIS_313-11732 & Qing Zao 3 & Indica \\
\hline IRIS_313-11733 & Shuang Bai Ai 2 & Indica \\
\hline IRIS_313-11734 & Si Chao 1 & Indica \\
\hline IRIS_313-11735 & Yi Li Zhong & japonica \\
\hline IRIS_313-11744 & Ai Jiao Ao Fan Zi & Indica \\
\hline IRIS_313-11745 & An Fu Zhan & Indica \\
\hline IRIS_313-11746 & E 2070 & Indica \\
\hline IRIS_313-11747 & E 4197 & japonica \\
\hline IRIS_313-11748 & Gao Jiao Ying Gan Zhan & Indica \\
\hline
\end{tabular}


Table S2. Putative phosphorylation sites in rice GPAT genes predicted in both NetPhos 3.1 and GPS 3.0

\begin{tabular}{|c|c|c|c|c|c|}
\hline & \multicolumn{3}{|c|}{ GPS 5.0} & \multicolumn{2}{|c|}{ NetPhos 3.1} \\
\hline Gene ID & Position & GPS 5.0 (kinase) & Score & NetPhos 3.1(Kinase) & Residue phosphorylated \\
\hline \multirow[t]{4}{*}{ OsGPAT1 } & 28 & AGC/AKT/AKT1 & 5.002 & 0.834 PKA & Serine $(S)$ \\
\hline & 170 & AGC/AKT/AKT1 & 1.263 & 0.959 unsp & Serine $(S)$ \\
\hline & 451 & AGC/AKT/AKT1 & 0.616 & 0.968 unsp & Serine $(S)$ \\
\hline & 497 & AGC/AKT/AKT1 & 0.446 & 0.983 unsp & Serine $(S)$ \\
\hline OsGPAT2 & 173 & AGC/AKT/AKT1 & 3.205 & 0.975 unsp & Serine $(S)$ \\
\hline \multirow{5}{*}{ OsGPAT3 } & 42 & AGC/AKT/AKT1 & 3.919 & 0.993 unsp & Serine $(S)$ \\
\hline & 43 & AGC/AKT/AKT1 & 0.91 & 0.917 unsp & Serine $(S)$ \\
\hline & 102 & AGC/AKT/AKT1 & 0.435 & 0.853 unsp & Threonine(T) \\
\hline & 144 & AGC/AKT/AKT1 & 0.662 & 0.989 unsp & Serine $(S)$ \\
\hline & 254 & AGC/AKT/AKT1 & 1.457 & 0.977 unsp & Serine $(S)$ \\
\hline \multirow[t]{4}{*}{ OsGPAT4 } & 37 & AGC/AKT/AKT1 & 1.838 & 0.964 unsp & Serine $(S)$ \\
\hline & 207 & AGC/AKT/AKT1 & 2.327 & $0.699 \mathrm{PKB}$ & Threonine(T) \\
\hline & 267 & AGC/AKT/AKT1 & 0.857 & 0.995 unsp & Serine $(S)$ \\
\hline & 438 & AGC/AKT/AKT1 & 0.951 & 0.968 unsp & Serine $(S)$ \\
\hline OsGPAT5 & 82 & AGC/AKT/AKT1 & 1.127 & 0.803 unsp & Threonine(T) \\
\hline \multirow[t]{5}{*}{ OsGPAT6 } & 35 & AGC/AKT/AKT1 & 2.015 & 0.686 PKG & Serine $(S)$ \\
\hline & 216 & AGC/AKT/AKT1 & 4.289 & 0.803 unsp & Serine $(S)$ \\
\hline & 324 & AGC/AKT/AKT1 & 1.68 & 0.779 PKA & Serine $(S)$ \\
\hline & 478 & AGC/AKT/AKT1 & 3.246 & 0.772 unsp & Serine $(S)$ \\
\hline & 489 & AGC/AKT/AKT1 & 4.461 & 0.874 unsp & Serine $(S)$ \\
\hline \multirow[t]{2}{*}{ OsGPAT7 } & 56 & AGC/AKT/AKT1 & 0.733 & 0.975 unsp & Threonine(T) \\
\hline & 61 & AGC/AKT/AKT1 & 1.095 & 0.919 unsp & Serine $(S)$ \\
\hline \multirow[t]{4}{*}{ OsGPATS } & 168 & AGC/AKT/AKT1 & 2.299 & 0.822 unsp & Threonine(T) \\
\hline & 283 & AGC/AKT/AKT1 & 1.257 & 0.819 PKC & Threonine(T) \\
\hline & 412 & AGC/AKT/AKT1 & 1.166 & 0.801 unsp & Threonine(T) \\
\hline & 19 & AGC/AKT/AKT1 & 1.897 & 0.500 EGFR & Tyrosine $(\mathrm{Y})$ \\
\hline \multirow[t]{2}{*}{ OsGPAT9 } & 75 & AGC/AKT/AKT1 & 1.17 & 0.661 unsp & Serine $(S)$ \\
\hline & 343 & AGC/AKT/AKT1 & 0.557 & 0.632 unsp & Serine $(S)$ \\
\hline \multirow[t]{3}{*}{ OsGPAT 10} & 23 & AGC/AKT/AKT1 & 3.744 & 0.678 PKA & Serine $(S)$ \\
\hline & 43 & AGC/AKT/AKT1 & 1.2 & 0.995 unsp & Serine $(S)$ \\
\hline & 436 & AGC/AKT/AKT1 & 0.44 & 0.989 unsp & Serine $(S)$ \\
\hline \multirow[t]{5}{*}{ OsGPAT 11 } & 12 & AGC/AKT/AKT1 & 0.48 & $0.701 \quad \mathrm{PKC}$ & Serine $(S)$ \\
\hline & 196 & AGC/AKT/AKT1 & 1.374 & 0.772 unsp & Threonine(T) \\
\hline & 233 & AGC/AKT/AKT1 & 2.784 & 0.832 PKA & Serine $(S)$ \\
\hline & 277 & AGC/AKT/AKT1 & 1.982 & 0.996 unsp & Serine $(S)$ \\
\hline & 292 & AGC/AKT/AKT1 & 4.433 & 0.725 unsp & Serine $(S)$ \\
\hline OsGPAT 12 & 67 & AGC/AKT/AKT1 & 1.015 & 0.705 unsp & Serine $(S)$ \\
\hline \multirow{5}{*}{ OsGPAT 13} & 34 & AGC/AKT/AKT1 & 1.277 & 0.984 unsp & Serine $(S)$ \\
\hline & 334 & AGC/AKT/AKT1 & 2.973 & 0.996 unsp & Serine $(S)$ \\
\hline & 423 & AGC/AKT/AKT1 & 0.554 & 0.968 unsp & Serine $(S)$ \\
\hline & 241 & AGC/AKT/AKT1 & 1.828 & 0.972 unsp & Tyrosine $(\mathrm{Y})$ \\
\hline & 521 & AGC/AKT/AKT1 & 2.286 & 0.989 unsp & Tyrosine $(\mathrm{Y})$ \\
\hline \multirow[t]{3}{*}{ OsGPAT 14} & 245 & AGC/AKT/AKT1 & 0.726 & 0.991 unsp & Serine $(S)$ \\
\hline & 314 & AGC/AKT/AKT1 & 0.932 & 0.996 unsp & Serine $(S)$ \\
\hline & 394 & AGC/AKT/AKT1 & 0.961 & 0.590 DNAPK & Serine $(S)$ \\
\hline \multirow[t]{5}{*}{ OsGPAT 15} & 115 & AGC/AKT/AKT1 & 0.533 & 0.548 PKA & Serine $(S)$ \\
\hline & 272 & AGC/AKT/AKT1 & 1.904 & 0.688 PKA & Serine $(S)$ \\
\hline & 393 & AGC/AKT/AKT1 & 1.388 & 0.835 PKA & Serine $(S)$ \\
\hline & 458 & AGC/AKT/AKT1 & 0.46 & 0.960 unsp & Serine $(S)$ \\
\hline & 476 & AGC/AKT/AKT1 & 0.781 & 0.527 CKI & Serine $(S)$ \\
\hline OsGPAT 16 & 18 & AGC/AKT/AKT1 & 3.423 & 0.995 unsp & Serine $(S)$ \\
\hline
\end{tabular}




\begin{tabular}{|c|c|c|c|c|c|}
\hline & 159 & AGC/AKT/AKT1 & 4.659 & 0.909 unsp & Threonine(T) \\
\hline & 195 & AGC/AKT/AKT1 & 1.719 & 0.995 unsp & Serine $(S)$ \\
\hline & 392 & AGC/AKT/AKT1 & 0.412 & 0.632 unsp & Serine $(S)$ \\
\hline \multirow[t]{4}{*}{ OsGPAT 17} & 265 & AGC/AKT/AKT1 & 2.07 & 0.61 PKC & Threonine(T) \\
\hline & 80 & AGC/AKT/AKT1 & 1.012 & 0.981 unsp & Serine $(S)$ \\
\hline & 397 & AGC/AKT/AKT1 & 1.947 & 0.711 PKC & Serine $(S)$ \\
\hline & 11 & AGC/AKT/AKT1 & 2.454 & 0.61 unsp & Tyrosine (Y) \\
\hline \multirow[t]{4}{*}{ OsGPAT 18} & 7 & AGC/AKT/AKT1 & 2.052 & 0.987 unsp & Serine $(S)$ \\
\hline & 74 & AGC/AKT/AKT1 & 2.432 & 0.998 unsp & Serine $(S)$ \\
\hline & 83 & AGC/AKT/AKT1 & 0.531 & 0.705 unsp & Tyrosine $(\mathrm{Y})$ \\
\hline & 532 & AGC/AKT/AKT1 & 0.501 & 0.696 PKC & Serine $(S)$ \\
\hline \multirow[t]{4}{*}{ OsGPAT 19} & 348 & AGC/AKT/AKT1 & 4.263 & 0.998 unsp & Serine $(S)$ \\
\hline & 154 & AGC/AKT/AKT1 & 1.459 & 0.912 unsp & Serine $(S)$ \\
\hline & 284 & AGC/AKT/AKT1 & 1.462 & 0.732 PKA & Serine $(S)$ \\
\hline & 362 & AGC/AKT/AKT1 & 0.991 & 0.742 PKC & Serine $(S)$ \\
\hline \multirow[t]{4}{*}{ OsGPAT 20} & 10 & AGC/AKT/AKT1 & 2.668 & 0.794 PKA & Serine $(S)$ \\
\hline & 70 & AGC/AKT/AKT1 & 0.599 & 0.917 unsp & Threonine(T) \\
\hline & 293 & AGC/AKT/AKT1 & 1.168 & 0.776 unsp & Threonine(T) \\
\hline & 376 & AGC/AKT/AKT1 & 0.768 & 0.968 unsp & Serine $(S)$ \\
\hline \multirow[t]{2}{*}{ OsGPAT 21} & 65 & AGC/AKT/AKT1 & 5.53 & 0.988 unsp & Threonine(T) \\
\hline & 219 & AGC/AKT/AKT1 & 0.983 & 0.974 unsp & Serine $(S)$ \\
\hline \multirow[t]{4}{*}{ OsGPAT 22} & 343 & AGC/AKT/AKT1 & 2.76 & 0.700 PKA & Serine $(S)$ \\
\hline & 10 & AGC/AKT/AKT1 & 1.458 & $0.770 \mathrm{PKB}$ & Threonine(T) \\
\hline & 256 & AGC/AKT/AKT1 & 0.588 & 0.641 PKA & Serine $(S)$ \\
\hline & 368 & AGC/AKT/AKT1 & 0.599 & 0.968 unsp & Serine $(S)$ \\
\hline \multirow[t]{5}{*}{ OsGPAT 23} & 268 & AGC/AKT/AKT1 & 1.005 & 0.835 unsp & Threonine(T) \\
\hline & 270 & AGC/AKT/AKT1 & 0.736 & 0.988 unsp & Serine $(S)$ \\
\hline & 282 & AGC/AKT/AKT1 & 1.173 & 0.802 PKA & Serine $(S)$ \\
\hline & 415 & AGC/AKT/AKT1 & 0.549 & 0.882 unsp & Serine $(S)$ \\
\hline & 418 & AGC/AKT/AKT1 & 0.873 & 0.908 unsp & Serine $(S)$ \\
\hline \multirow[t]{4}{*}{ OsGPAT 24} & 38 & AGC/AKT/AKT1 & 2.222 & 0.982 unsp & Serine $(S)$ \\
\hline & 121 & AGC/AKT/AKT1 & 0.474 & 0.954 unsp & Serine $(S)$ \\
\hline & 362 & AGC/AKT/AKT1 & 2.503 & 0.950 unsp & Serine $(S)$ \\
\hline & 363 & AGC/AKT/AKT1 & 6.311 & 0.998 unsp & Serine $(S)$ \\
\hline \multirow[t]{9}{*}{ OsGPAT 25} & 7 & AGC/AKT/AKT1 & 1.334 & 0.951 unsp & Serine $(S)$ \\
\hline & 12 & AGC/AKT/AKT1 & 1.545 & $0.550 \mathrm{PKC}$ & Serine $(S)$ \\
\hline & 29 & AGC/AKT/AKT1 & 1.819 & 0.996 unsp & Serine $(S)$ \\
\hline & 249 & AGC/AKT/AKT1 & 0.435 & 0.706 PKA & Serine $(S)$ \\
\hline & 365 & AGC/AKT/AKT1 & 1.437 & 0.991 unsp & Serine $(S)$ \\
\hline & 390 & AGC/AKT/AKT1 & 1.017 & 0.998 unsp & Serine $(S)$ \\
\hline & 423 & AGC/AKT/AKT1 & 0.68 & 0.873 unsp & Serine $(S)$ \\
\hline & 489 & AGC/AKT/AKT1 & 1.603 & 0.880 unsp & Serine $(S)$ \\
\hline & 535 & AGC/AKT/AKT1 & 1.174 & 0.952 unsp & Serine $(S)$ \\
\hline \multirow[t]{6}{*}{ OsGPAT 26} & 65 & AGC/AKT/AKT1 & 2.422 & 0.980 unsp & Serine $(S)$ \\
\hline & 156 & AGC/AKT/AKT1 & 3.563 & 0.991 unsp & Serine $(S)$ \\
\hline & 157 & AGC/AKT/AKT1 & 3.454 & 0.998 unsp & Serine $(S)$ \\
\hline & 353 & AGC/AKT/AKT1 & 2.858 & 0.956 unsp & Threonine(T) \\
\hline & 387 & AGC/AKT/AKT1 & 0.996 & 0.991 unsp & Serine $(S)$ \\
\hline & 445 & AGC/AKT/AKT1 & 1.17 & 0.968 unsp & Serine $(S)$ \\
\hline
\end{tabular}


Table S3. Putative methylation sites of rice GPAT genes predicted by PSSMe and iMethyl-PseAAAC toolss

\begin{tabular}{|c|c|c|c|c|}
\hline \multirow[b]{2}{*}{ Gene Id } & \multirow[b]{2}{*}{ Position } & \multicolumn{2}{|l|}{ PSSMe } & \multirow{2}{*}{$\begin{array}{c}\text { iMethyl-PseAAC } \\
\text { Methylation position site }\end{array}$} \\
\hline & & Flanking residue & SVM Probability & \\
\hline \multirow[t]{4}{*}{ OsGPAT1 } & 292 & PPGGECGGV-K-PLVFHDGRL & 0.80542 & 292 \\
\hline & 483 & HGTSTTPAA-K-WMDPFYFMM & 0.89011 & 483 \\
\hline & 495 & DPFYFMMNP-K-PSYRVEFLP & 0.8908 & 495 \\
\hline & 545 & ELTGMTRKD-K-YMMLAGNEG & 0.62344 & 545 \\
\hline \multirow[t]{7}{*}{ OsGPAT2 } & 44 & MVMVCFFGL-K-EKKVARVAR & 0.7091 & 44 \\
\hline & 46 & MVCFFGLKE-K-KVARVARAA & 0.56021 & 46 \\
\hline & 91 & SRVIPRVMV-K-PFLEDYLGV & 0.53274 & 91 \\
\hline & 182 & SPLPRDQYP-K-PLVFHDGRL & 0.73319 & 182 \\
\hline & 251 & VINSPVGQA-K-ADHPRNPKG & 0.64146 & 251 \\
\hline & 259 & AKADHPRNP-K-GHLYVCNHR & 0.57032 & 259 \\
\hline & 438 & ECTKFTREN-K-YLALAGNRG & 0.54876 & 438 \\
\hline \multirow[t]{2}{*}{ OsGPAT3 } & 175 & FHGTTAGGW-K-LLDPLYLLM & 0.75545 & 175 \\
\hline & 265 & TRTALTRRD-K-YLALTGNDG & 0.5 & 265 \\
\hline \multirow[t]{5}{*}{ OsGPAT4 } & 9 & OMVLPTILP-K-IAAHWLFTF & 0.6076 & 9 \\
\hline & 65 & TVVFPDAAD-K-AVVFGFDGA & 0.53222 & 65 \\
\hline & 145 & DLVARAVLP-K-FYMEGLNAQ & 0.86769 & 145 \\
\hline & 280 & ARLPRDRYP-K-PLIFHDGRL & 0.78427 & 280 \\
\hline & 325 & ISIGILLPY-K-ISFGAGALF & 0.72122 & 325 \\
\hline \multirow[t]{6}{*}{ OsGPAT5 } & 147 & LRKGYLGYI-K-YILKSSLMK & 0.66405 & 147 \\
\hline & 151 & YLGYIKYIL-K-SSLMKLPVF & 0.81681 & 151 \\
\hline & 175 & IFEFIPVER-K-WEIDEAIIQ & 0.61441 & 175 \\
\hline & 191 & IIQNKLSAF-K-DPRDPLWLA & 0.69038 & 191 \\
\hline & 229 & ASEHGLPIL-K-NVLLPKTKG & 0.86028 & 229 \\
\hline & 362 & LSLFSSVWF-K-VYVLLSCAY & 0.69629 & 362 \\
\hline \multirow[t]{8}{*}{ OsGPAT6 } & 102 & EMVARSVLP-K-FYAEDVHPE & 0.54789 & 102 \\
\hline & 121 & SWRVFNSFG-K-RYIITASPR & 0.64426 & 121 \\
\hline & 155 & VVGTELEVG-K-NGKATGFMV & 0.89347 & 155 \\
\hline & 277 & PERIVFYTY-K-LMGIRLIVK & 0.83839 & 277 \\
\hline & 286 & KLMGIRLIV-K-GNPPPPPKK & 0.9418 & 286 \\
\hline & 294 & VKGNPPPPP-K-KGHPGVLFV & 0.93903 & 294 \\
\hline & 295 & KGNPPPPPK-K-GHPGVLFVC & 0.95441 & 295 \\
\hline & 414 & FHGSTVRGF-K-LMDPYFFFM & 0.71944 & 414 \\
\hline \multirow[t]{6}{*}{ OsGPAT7 } & 97 & SSSALRFYR-K-KVGKEVDGI & 0.63366 & 97 \\
\hline & 98 & SSALRFYRK-K-VGKEVDGIE & 0.78182 & 98 \\
\hline & 213 & ILSTFFRSV-K-VLPVSRGDG & 0.62596 & 213 \\
\hline & 255 & EGSRSKDGG-K-TVAPAKRGV & 0.67056 & 255 \\
\hline & 293 & GMQDIMPVG-K-RIPRAGKRV & 0.81575 & 293 \\
\hline & 404 & EPSDVQEPL-K-KAKPVLHLE & 0.62035 & 404 \\
\hline \multirow[t]{3}{*}{ OsGPAT8 } & 212 & SDHDFMAIC-K-EAYMVPKNK & 0.736 & 212 \\
\hline & 219 & ICKEAYMVP-K-NKRAPRAAA & 0.65467 & 219 \\
\hline & 221 & KEAYMVPKN-K-RAPRAAADE & 0.5874 & 221 \\
\hline \multirow[t]{4}{*}{ OsGPAT9 } & 183 & RPVPREELP-K-PVVFHDGRL & & 183 \\
\hline & 195 & VFHDGRLVQ-K-PSPALALLT & & 195 \\
\hline & 374 & FHGTTARGW-K-ALDPFYFFM & & 374 \\
\hline & 440 & SYECTSFTR-K-DKYRALAGN & & 440 \\
\hline \multirow[t]{3}{*}{ OsGPAT 10} & 170 & PREMVEPFL-K-EYLAVDAVV & 0.50755 & 170 \\
\hline & 467 & FHGTTAGGW-K-MLDPFFLLM & 0.78342 & 467 \\
\hline & 539 & NDGVVANNN-K-SNOOOOOOO & 0.51865 & 539 \\
\hline OsGPAT11 & 99 & LMWILGNPI-K-LEGMENLNT & 0.90227 & 99 \\
\hline
\end{tabular}




\begin{tabular}{|c|c|c|c|c|}
\hline & 138 & APTGTVGIA-K-KEIIWYPLF & 0.86211 & 138 \\
\hline & 139 & PTGTVGIAK-K-EIIWYPLFG & 0.78888 & 139 \\
\hline & 203 & SKTGRLLPF-K-KGFVHTALQ & 0.60463 & 203 \\
\hline & 279 & YADSLPDSQ-K-PLEPVNTGK & 0.82815 & 279 \\
\hline \multirow[t]{8}{*}{ OsGPAT13 } & 3 & OOOOOOOMA-K-KPCEFPTAV & 0.91101 & 3 \\
\hline & 4 & OOOOOOMAK-K-PCEFPTAVL & 0.79813 & 4 \\
\hline & 47 & TGAAIPPAD-K-LHNQTVMID & 0.91236 & 47 \\
\hline & 119 & MVSFFGLPE-K-EVVRIGKAV & 0.87515 & 119 \\
\hline & 126 & PEKEVVRIG-K-AVLPKFFLE & 0.73036 & 126 \\
\hline & 131 & VRIGKAVLP-K-FFLEGMAME & 0.51934 & 131 \\
\hline & 149 & EGLEVVRNA-K-KVVVFSPLF & 0.76715 & 149 \\
\hline & 228 & AVGLAGVGS-K-MHHLFSRYC & 0.69155 & 228 \\
\hline \multirow[t]{3}{*}{ OsGPAT 14} & 220 & SSSFPSFVA-K-RSVARLPMV & 0.6271 & 220 \\
\hline & 311 & KPVILRYPY-K-RFSPAWDSM & 0.65331 & 311 \\
\hline & 356 & PSEQEKEDP-K-LYANNVRKL & 0.55487 & 356 \\
\hline \multirow[t]{4}{*}{ OsGPAT15 } & 5 & OOOOOMSPF-K-PIEQCSTEG & 0.90928 & 5 \\
\hline & 172 & KPGVLIREH-K-RNAVVREFG & 0.51898 & 172 \\
\hline & 203 & SDFDFMAIC-K-DAYVVTTSR & 0.84008 & 203 \\
\hline & 423 & FHGSTARGF-K-GMDPYFFFM & 0.58306 & 423 \\
\hline \multirow[t]{3}{*}{ OsGPAT16 } & 205 & ATRSFLPFC-K-KQLRPPFCE & 0.65357 & 205 \\
\hline & 206 & TRSFLPFCK-K-QLRPPFCED & 0.59545 & 206 \\
\hline & 423 & FHPTTARGW-K-AMDPIFFFM & 0.5238 & 423 \\
\hline \multirow[t]{6}{*}{ OsGPAT 17} & 101 & WPFLFEKIN- K-TKFVFSGET & 0.77231 & 101 \\
\hline & 146 & LRKGRLQCI- K-YILKKSLMK & 0.60963 & 146 \\
\hline & 150 & RLQCIKYIL- K-KSLMKLPIF & 0.77819 & 150 \\
\hline & 151 & LQCIKYILK- K-SLMKLPIFN & 0.826 & 151 \\
\hline & 174 & IIEFIPVER- K-WEVDEPLIR & 0.69241 & 174 \\
\hline & 361 & LTLFSSVWF- K-IFVAFSSAF & 0.7578 & 361 \\
\hline \multirow[t]{4}{*}{ OsGPAT 18} & 172 & SFGYHWIRR-K-GKPAPRELA & 0.74776 & 172 \\
\hline & 311 & NISLGKLMF-K-MFTQFHNFM & 0.58198 & 311 \\
\hline & 334 & LPVVYPPEI-K-QENALHFAE & 0.84077 & 334 \\
\hline & 447 & IFQYFDFEA-K-ESITFRQFL & 0.52331 & 447 \\
\hline \multirow[t]{11}{*}{ OsGPAT 19} & 118 & AFFPVHFLL-K-GQKMRSKIE & 0.61251 & 118 \\
\hline & 147 & FVASWTGVI-K-YHGPRPSTR & 0.76356 & 147 \\
\hline & 186 & MTAFAVIMQ-K-HPGWVGFIQ & 0.60809 & 186 \\
\hline & 213 & CIWFNRNDL-K-DREVVAKKL & 0.63237 & 213 \\
\hline & 221 & LKDREVVAK-K-LRDHVQHPD & 0.66122 & 221 \\
\hline & 252 & VNNQYTVMF-K-KGAFELGCA & 0.65937 & 252 \\
\hline & 253 & NNQYTVMFK-K-GAFELGCAV & 0.79717 & 253 \\
\hline & 271 & VCPIAIKYN-K-IFVDAFWNS & 0.72962 & 271 \\
\hline & 281 & IFVDAFWNS-K-KQSFTMHLV & 0.78375 & 281 \\
\hline & 334 & DMIAARAGL-K-KVPWDGYLK & 0.60078 & 334 \\
\hline & 343 & KKVPWDGYL-K-HNRPSPKHT & 0.68569 & 343 \\
\hline \multirow[t]{4}{*}{ OsGPAT21 } & 193 & LGRCFKFIS- K-TSIFMFPII & 0.91653 & 193 \\
\hline & 235 & LKRCVDLVK-K-GASVFFFPE & 0.70259 & 235 \\
\hline & 252 & PEGTRSKDG- K-LGAFKRGAF & 0.62248 & 252 \\
\hline & 281 & IPITLLGTG- K-LMPSGMEGI & 0.86417 & 281 \\
\hline \multirow[t]{5}{*}{ OsGPAT23 } & 37 & RRGALRLEA- K-AAWRPAARG & 0.62753 & 37 \\
\hline & 85 & ILHIRKEVE- K-GKLPADVAA & 0.72125 & 85 \\
\hline & 145 & NPFTFPPYH- K-AVREPFDYY & 0.89176 & 145 \\
\hline & 345 & CYEVMPPPQ- K-VEKEIGEQR & 0.78865 & 345 \\
\hline & 348 & VMPPPQKVE- K-EIGEQRVIS & 0.5 & 348 \\
\hline \multirow[t]{2}{*}{ OsGPAT24 } & 39 & LFLSIRPFS- K-SLYRRINRF & 0.89326 & 39 \\
\hline & 146 & YLFLERSWA- K-DEKTLKWGL & 0.62846 & 146 \\
\hline
\end{tabular}




\begin{tabular}{|l|c|c|c|c|}
\hline & 149 & LERSWAKDE- K-TLKWGLQRL & 0.5 & 149 \\
\hline & 159 & TLKWGLQRL-K-DFPRPFWLA & 0.72151 & 159 \\
\hline & 180 & VEGTRFTPA-K-LLAAQEYAV & 0.78081 & 230 \\
\hline & 230 & IYDTTVIIP- K-DSPQPTMLR & 0.63331 & 253 \\
\hline & 253 & QSSVVHVRM-K-RHAMSEMPK & 0.57787 & 278 \\
\hline & 278 & KWCKDIFVA-K-DALLDKHLA & 0.80689 & 333 \\
\hline OsGPAT25 & 333 & LWTQLLSTW-K-GVGFTGLGL & 0.72294 & 4 \\
\hline & 3 & OOOOOOOMP-K-KKLSHRLFS & 0.65634 & 5 \\
\hline & 4 & OOOOOOMPK-K-KLSHRLFSA & 0.73619 & 43 \\
\hline & 5 & OOOOOMPKK-K-LSHRLFSAL & 0.55845 & 110 \\
\hline & 43 & TLPHPSLLH- K-SSSSFPPME & 0.73673 & 134 \\
\hline & 110 & CVMGSDMAL-K-VMAMVSFCG & 0.80442 & 184 \\
\hline & 134 & FRAGRAVLP-K-WFLEDVGEE & 0.57445 & 255 \\
\hline & 184 & VEVVSGREM-K-VIWGFFTGI & 0.83552 & 258 \\
\hline & 255 & ARWSALPRD-K-YPKPMVFHD & 0.84497 & 319 \\
\hline & 258 & SALPRDKYP-K-PMVFHDGRL & 0.75166 & 514 \\
\hline & 319 & AATGLSWRL-K-GEAPTPLAG & 0.64871 & 3 \\
\hline & 514 & GYRCTMLTR-K-DKYLMLAGN & 0.5 & 5 \\
\hline
\end{tabular}


Table S4. Putative uiquitylation sites of rice GPAT genes predicted by BDM-PUB and Ub-Pred

\begin{tabular}{|c|c|c|c|c|c|c|}
\hline & \multicolumn{3}{|c|}{ BDM-PUB } & \multicolumn{3}{|c|}{ Ub-Pred } \\
\hline Gene ID & Position & score & Threshold & Position & score & Ubiquitinated \\
\hline OsGPAT2 & 169 & 0.4 & 0.3 & 169 & 0.62 & Yes-Low confidence \\
\hline \multirow[t]{3}{*}{ OsGPAT4 } & 42 & 0.47 & 0.3 & 42 & 0.68 & Yes-Low confidence \\
\hline & 65 & 1.44 & 0.3 & 65 & 0.65 & Yes-Medium confidence \\
\hline & 544 & 3.93 & 0.3 & 544 & 0.65 & Yes-Low confidence \\
\hline \multirow[t]{2}{*}{ OsGPAT5 } & 18 & 1.3 & 0.3 & 18 & 0.69 & Yes-Low confidence \\
\hline & 396 & 4.12 & 0.3 & 396 & 0.64 & Yes-Low confidence \\
\hline OsGPAT 6 & 7 & 1.12 & 0.3 & 7 & 0.68 & Yes-Low confidence \\
\hline \multirow[t]{3}{*}{ OsGPAT7 } & 251 & 2.26 & 0.3 & 251 & 0.62 & Yes-Low confidence \\
\hline & 358 & 0.55 & 0.3 & 358 & 0.67 & Yes-Low confidence \\
\hline & 429 & 0.48 & 0.3 & 429 & 0.9 & Yes-High confidence \\
\hline OsGPAT\& & 502 & 4.16 & 0.3 & 502 & 0.85 & Yes-High confidence \\
\hline \multirow{3}{*}{ OsGPAT9 } & 456 & 0.46 & 0.3 & 456 & 0.62 & Yes-Low confidence \\
\hline & 462 & 1.76 & 0.3 & 462 & 0.67 & Yes-Low confidence \\
\hline & 463 & 1.49 & 0.3 & 463 & 0.73 & Yes-Medium confidence \\
\hline \multirow[t]{3}{*}{ OsGPAT 10} & 57 & 0.81 & 0.3 & 57 & 0.62 & Yes-Low confidence \\
\hline & 213 & 0.49 & 0.3 & 213 & 0.65 & Yes-Low confidence \\
\hline & 539 & 2.33 & 0.3 & 539 & 0.71 & Yes-Medium confidence \\
\hline OsGPAT 12 & 45 & 2.47 & 0.3 & 45 & 0.46 & Yes-Medium confidence \\
\hline \multirow[t]{2}{*}{ OsGPAT 13} & 47 & 2.42 & 0.3 & 47 & 0.67 & Yes-Low confidence \\
\hline & 532 & 3.26 & 0.3 & 532 & 0.64 & Yes-Low confidence \\
\hline \multirow[t]{4}{*}{ OsGPAT 14} & 32 & 1.93 & 0.3 & 32 & 0.95 & Yes-High confidence \\
\hline & 188 & 0.71 & 0.3 & 188 & 0.79 & Yes-Medium confidence \\
\hline & 246 & 1.02 & 0.3 & 246 & 0.66 & Yes-Low confidence \\
\hline & 402 & 3.4 & 0.3 & 402 & 0.81 & Yes-Medium confidence \\
\hline \multirow[t]{3}{*}{ OsGPAT 15} & 5 & 1.45 & 0.3 & 5 & 0.8 & Yes-Medium confidence \\
\hline & 348 & 2.98 & 0.3 & 348 & 0.65 & Yes-Low confidence \\
\hline & 457 & 0.81 & 0.3 & 457 & 0.81 & Yes-Medium confidence \\
\hline OsGPAT 17 & 395 & 4.72 & 0.3 & 395 & 0.62 & Yes-Low confidence \\
\hline OsGPAT 18 & 376 & 0.52 & 0.3 & 376 & 0.62 & Yes-Low confidence \\
\hline OsGPAT 20 & 208 & 1.13 & 0.3 & 208 & 0.75 & Yes-Medium confidence \\
\hline OsGPAT 22 & 63 & 1.41 & 0.3 & 208 & 0.64 & Yes-Low confidence \\
\hline OsGPAT 24 & 262 & 1.05 & 0.3 & 262 & 0.67 & Yes-Low confidence \\
\hline \multirow[t]{2}{*}{ OsGPAT 25} & 43 & 1.2 & 0.3 & 43 & 0.83 & Yes-Medium confidence \\
\hline & 53 & 2.7 & 0.3 & 53 & 0.85 & Yes-High confidence \\
\hline \multirow[t]{2}{*}{ OsGPAT 26} & 48 & 0.83 & 0.3 & 48 & 0.78 & Yes-Medium confidence \\
\hline & 508 & 2.7 & 0.3 & 508 & 0.73 & Yes-Medium confidence \\
\hline
\end{tabular}


Table S5. Highly conserved amino acid positions in Post translation modification sites

\begin{tabular}{|c|c|c|c|}
\hline Gene ID & Methylation & Phosphorylation & Ubiquitylation \\
\hline \multirow[t]{2}{*}{ OsGPAT1 } & $\mathrm{K} 483$ & & \\
\hline & K545 & & \\
\hline \multirow[t]{2}{*}{ OsGPAT2 } & K91 & & \\
\hline & K438 & & \\
\hline OsGPAT4 & K145 & & K544 \\
\hline OsGPAT5 & K151 & & K396 \\
\hline \multirow[t]{2}{*}{ OsGPAT6 } & K102 & & \\
\hline & K414 & & \\
\hline \multirow[t]{4}{*}{ OsGPAT7 } & K251 & & \\
\hline & K97 & & \\
\hline & K213 & & \\
\hline & K404 & & \\
\hline OsGPAT8 & K212 & & \\
\hline OsGPAT9 & K374 & S343 & \\
\hline OsGPAT 10 & K467 & & \\
\hline \multirow[t]{3}{*}{ OsGPAT 11} & K138 & & \\
\hline & K139 & & \\
\hline & K203 & & \\
\hline Os GPAT 12 & & & $\mathrm{~K} 45$ \\
\hline \multirow[t]{2}{*}{ OsGPAT 13} & A126 & Y521 & \\
\hline & K131 & $S 423$ & \\
\hline \multirow[t]{2}{*}{ OsGPAT 14} & K220 & S245 & K246 \\
\hline & & S314 & K402 \\
\hline Os GPAT 15 & K203 & S393 & \\
\hline \multirow[t]{2}{*}{ OsGPAT16 } & K423 & & \\
\hline & & S392 & \\
\hline \multirow[t]{2}{*}{ OsGPAT 17} & K150 & & K395 \\
\hline & K151 & & \\
\hline Os GPAT 18 & K311 & & \\
\hline \multirow[t]{3}{*}{ OsGPAT 19} & K252 & & \\
\hline & K253 & & \\
\hline & K343 & & \\
\hline OsGPAT20 & & S376 & \\
\hline OsGPAT21 & K193 & & \\
\hline OsGPAT22 & & S368 & \\
\hline OsGPAT23 & K348 & & \\
\hline \multirow[t]{4}{*}{ OsGPAT24 } & K39 & & \\
\hline & K146 & & \\
\hline & K180 & & \\
\hline & $\mathrm{k} 278$ & & \\
\hline \multirow[t]{2}{*}{ Os GPAT25 } & K134 & S365 & \\
\hline & K514 & $S 423$ & \\
\hline \multirow[t]{2}{*}{ OsGPAT26 } & & S387 & \\
\hline & & $S 445$ & \\
\hline
\end{tabular}


Table S6. List of the 26 rice GPAT genes and their detailed information including gene ID, locus positions, gene and protein size taken from a previous study. OsGPAT denotes rice GPAT genes

\begin{tabular}{|c|c|c|c|c|}
\hline Gene Name & Locus ID & Chromosomal position & Gene (bp) & Protein \\
\hline OsGPAT 1 & LOC_Os01g14900 & Chr01: 8347893-8351006 & 3114 & 571 \\
\hline OsGPAT2 & LOC_Os01g19390 & Chr01: $10972881-10969331$ & 3551 & 454 \\
\hline OsGPAT3 & LOC_Os01g22560 & Chr01: $12678141-12677086$ & 1056 & 265 \\
\hline OsGPAT4 & LOC_Os01g44069 & Chr01: $25257001-25263981$ & 6981 & 545 \\
\hline OsGPAT5 & LOC_Os01g 57360 & Chr01: $33158320-33154276$ & 4045 & 400 \\
\hline OsGPAT 6 & LOC_Os01g63580 & Chr01: $36866375-36863214$ & 3162 & 498 \\
\hline OsGPAT7 & LOC_Os01g70570 & Chr01: $40868356-40864908$ & 3449 & 508 \\
\hline OsGPAT 8 & LOC_Os02g02340 & Chr02: 778356 - 775494 & 2863 & 507 \\
\hline OsGPAT9 & LOC_Os03g52570 & Chr03: $30153564-30151748$ & 1817 & 468 \\
\hline OsGPAT 10 & LOC_Os03g61720 & Chr03: $34985196-34990759$ & 5564 & 542 \\
\hline OsGPAT 11 & LOC_Os04g53370 & Chr04:31786589- 31788246 & 1658 & 293 \\
\hline OsGPAT 12 & LOC_Os04g57150 & Chr04:34058149 - 34054300 & 3850 & 158 \\
\hline OsGPAT 13 & LOC_Os05g20100 & Chr05:11756381 - 11753281 & 3101 & 538 \\
\hline OsGPAT 14 & LOC_Os05g28960 & Chr05:16982192 - 16977760 & 4905 & 405 \\
\hline OsGPAT 15 & LOC_Os05g37600 & Chr05:22005196 - 22008976 & 3781 & 487 \\
\hline OsGPAT 16 & LOC_Os05g38350 & Chr05:22481303 - 22484545 & 3243 & 523 \\
\hline OsGPAT 17 & LOC_Os05g42270 & Chr05:24724324 - 24727260 & 2937 & 398 \\
\hline OsGPAT 18 & LOC_Os06g49790 & Chr06:30122819 - 30128027 & 5209 & 557 \\
\hline OsGPAT 19 & LOC_Os07g34730 & Chr07:20812544 - 20808033 & 4512 & 371 \\
\hline OsGPAT 20 & LOC_Os08g03700 & Chr08:1759198 - 1757711 & 1488 & 496 \\
\hline OsGPAT 21 & LOC_Os10g35390 & Chr10:18917046 - 18920354 & 3309 & 333 \\
\hline OsGPAT 22 & LOC_Os10g41070 & Chr10:22059229- 22060717 & 1489 & 480 \\
\hline OsGPAT 23 & LOC_Os10g42720 & Chr10:23044693 - 23050740 & 6048 & 428 \\
\hline OsGPAT 24 & LOC_Os11g41900 & Chr11:25206038 - 25199831 & 6208 & 375 \\
\hline OsGPAT 25 & LOC_Os11g45400 & Chr11:27486903 - 27490269 & 3367 & 544 \\
\hline OsGPAT 26 & LOC_Os12g37600 & Chr12:23091636 - 23094141 & 2506 & 559 \\
\hline
\end{tabular}

